\title{
HIGH-FIDELITY NONLINEAR ANALYSIS OF COMPRESSION-LOADED COMPOSITE SHELLS
}

\author{
Mark W. Hilburger ${ }^{*}$ and James H. Starnes, Jr. ${ }^{\dagger}$ \\ NASA Langley Research Center \\ Hampton, Virginia 23681-001
}

\begin{abstract}
The results of an experimental and analytical study of the effects of initial imperfections on the buckling and postbuckling response of unstiffened thinwalled compression-loaded graphite-epoxy cylindrical shells are presented. The shells considered in the study have four different shell-wall laminates and two different shell-radius-to-thickness ratios. The shell-wall laminates include two different orthotropic laminates and two different quasi-isotropic laminates. The shell-radius-to-thickness ratios include shell-radius-to-thickness ratios equal to 100 and 200 . The results identify the effects of traditional and nontraditional initial imperfections on the nonlinear response characteristics and buckling loads of the shells. The traditional imperfections include the geometric shell-wall mid-surface imperfections that are commonly discussed in the literature on thin shell buckling. The nontraditional imperfections include shell-wall thickness variations, local shell-wall ply-gaps associated with the fabrication process, shell-end geometric imperfections, nonuniform applied end loads, and variations in the boundary conditions including the effects of elastic boundary conditions. A high-fidelity nonlinear shell analysis procedure that accurately accounts for the effects of these traditional and nontraditional imperfections on the nonlinear response characteristics and buckling loads of the shells is described. The analysis procedure includes a nonlinear static analysis that predicts the stable response characteristics of the shells, and a nonlinear transient analysis that predicts the unstable response characteristics. The results of a local shell-wall stress analysis used to estimate failure stresses are also described.
\end{abstract}

\section{Introduction}

The increasing need to produce lighter-weight aerospace shell structures has led to the use of advanced material systems in new structural designs, and im-

\footnotetext{
Aerospace Engineer, Mechanics and Durability Branch. Member, AIAA

${ }^{\dagger}$ Senior Engineer, Structures and Materials Competency. Fellow, AlAA.

Copyright 92001 by the American Institute of Aeronautics and Astronautics, Ins. No copyright is asserted in the United States under Title 17, U. S. Code. The $U$. S. Government has a royaliy-free license to exercise all rights under the copyright claimed herein for Governmental Purposes. All other rights are reserved by the copyright owner.
}

proved design methods appropriate for these advanced material systems are needed. The high strength-toweight and high stiffness-to-weight ratios of advanced composite materials offer significant weight reduction potential for aerospace structures. In addition, the use of advanced composite materials allows the designer to tailor the stiffness properties of composite structures to obtain structurally efficient designs. Designers often use a design-level analysis procedure with empirical data to develop new structural designs for strength and buckling-critical structures. The traditional approach for designing thin-walled buckling-resistant isotropic shell structures is to predict the buckling load of the shell with a deterministic analysis, and then to reduce this predicted load with an empirical "knockdown" factor (e.g., Ref. 1). The empirical knockdown factor is intended to account for the difference between the predicted buckling load and the actual buckling load for the shell determined from tests. A linear bifurcation buckling analysis is often used for the design-level analysis, and this analysis is usually based on nominal structural dimensions and material properties of an idealized, geometrically perfect shell. The design knockdown factor used in the design of buckling-resistant shells is often based on the "lower bound" design recommendations reported in Ref. 1. This design philosophy can result in overly conservative designs for these structures, and it can potentially even result in unconservative designs if the empirical data are not representative of the design of interest. While it is generally recognized that initial geometric shell-wall imperfections are a major contributor to the discrepancy between the predicted shell buckling loads and the experimentally measured shell buckling loads (e.g., Refs. 2-6), the traditional sources of design knockdown factors do not include data or information related to the sensitivity of the response of a shell to various forms of imperfections. In addition, the traditional sources of design knockdown factors for predicting shell buckling loads do not include information for shell structures made from advanced composite materials. Recent studies (e.g., Refs. 7-11) have shown that traditional initial geometric shell-wall imperfections, and other nontraditional forms of imperfections or variations in geometric and material parameters, loading conditions, and boundary 
conditions can significantly affect the buckling load of a compression-loaded composite shell structure. Other recent studies (e.g., Ref. 12) have identified the effects of fabrication related anomalies on the buckling loads of metallic shells. The effects of these traditional and nontraditional classes of initial imperfections on the buckling of composite shells are generally not well understood by structural engineers and designers. Modern high-fidelity nonlinear analysis procedures (e.g., Ref. 13) offer the opportunity to improve some of the engineering approximations that are used in the design and analysis of shell structures, and to provide insight into the effects of traditional and nontraditional imperfections on the response of compression-loaded composite shell structures.

The present paper describes the results of an experimental and analytical study of the effects of traditional initial geometric shell-wall imperfections, and the effects of nontraditional initial imperfections and variations in other nontraditional geometric and material parameters, loading conditions, and boundary conditions on the buckling response of unstiffened thinwalled compression-loaded graphite-epoxy cylindrical shells. The results of four graphite-epoxy shells with different shell-wall laminates are presented. The shellwall laminates include two different orthotropic laminates and two quasi-isotropic laminates. The two orthotropic shells and one of the quasi-isotropic shells have shell-radius-to-thickness ratios equal to 200 , and the other quasi-isotropic shell has a shell-radius-tothickness ratio equal to 100 . Traditional shell-wall geometric imperfections and several nontraditional imperfections were measured, and representations of these imperfections have been included in nonlinear analyses of the shells. These nonlinear analyses were conducted with the geometrically nonlinear STAGS finite element analysis code (Ref. 13). The effects of initial geometric shell-wall imperfections, shell-wall thickness variations, shell-end geometric imperfections, nonuniform applied end loads, and variations in the boundary conditions, including the effects of elastic boundary conditions, on the buckling response of these thin-walled composite shells are discussed in the present paper. The effects of fabrication anomalies caused by small local gaps between adjacent pieces of the graphite-epoxy material in some of the shell-wall laminate plies are also discussed. The response of a quasi-isotropic shell with a shell-radius-to-thickness ratio equal to 100 is presented as an example of a thin-walled shell that has material failures before it buckles, and a local stress analysis of the shell wall is used to show that these local material failures can result in the overall failure of the shell. The results of the study are used to illustrate the significance of these nontraditional initial imperfec- tions on composite shell response characteristics. The nonlinear shell analysis procedure used to predict the nonlinear response and buckling loads of the shells is described, and the analysis results are compared with the experimental results. The use of this nonlinear shell analysis procedure for determining accurate, high-fidelity design knockdown factors for shell buckling and collapse, and for determining the effects of variations and uncertainties in shell geometric and material parameters on shell buckling loads is discussed.

\section{Test Specimens. Imperfection Measurements, and Test Apparatus and Tests}

\section{Test Specimens}

The specimens tested in this investigation were fabricated from 0.005 -in.-thick AS4/3502 graphite-epoxy preimpregnated unidirectional tape material made by Hercules, Inc. The nominal unidirectional lamina properties of a typical 0.005 -in.-thick ply with a fiber volume fraction of 0.62 are as follows: longitudinal modulus $E_{1}=19.5 \mathrm{Msi}$, transverse modulus $E_{2}=1.45$ Msi, in-plane shear modulus $G_{12}=0.813 \mathrm{Msi}$, and major Poisson's ratio $v_{12}=0.30$. The material was laid up on a 15.75-in.-diameter mandrel and cured in an autoclave to form four shells with different shell-wall laminates including an 8-ply axially stiff $\left[\mp 45 / 0_{2}\right]_{\mathrm{s}}$ laminate, an 8-ply circumferentially stiff $\left[\bar{\mp} 45 / 90_{2}\right]_{\mathrm{s}}$ laminate, an 8-ply quasi-isotropic [ $\lceil 45 / 0 / 90]_{s}$ laminate, and a 16 -ply quasi-isotropic $[\mp 45 / 0 / 90]_{2 s}$ laminate. The resulting four shells are referred to herein as shells or specimens $\mathrm{C} 1, \mathrm{C} 2, \mathrm{C} 3$ and $\mathrm{C} 4$, respectively. These specimens had a nominal length of $16.0 \mathrm{in}$. and a nominal radius of 8.0 in. The 8-ply and 16-ply specimens had a nominal shell-wall thickness of $0.04 \mathrm{in}$. and 0.08 in., respectively, and had shell-radius-to-thickness ratios of 200 and 100 , respectively. Both ends of the specimens were potted in an aluminum-filled epoxy resin to assure that the ends of the specimens did not fail prematurely during the test. The potting material extended approximately 1.0 inch along the length of the specimens at each end resulting in a test section that was approximately $14.0 \mathrm{in}$. long. The ends of the specimens were machined flat and parallel to assure proper load introduction during the tests. A photograph of a typical specimen and the specimen coordinate system used to represent the corresponding geometry is shown in Fig. 1. The shell length, test-section length, radius, and thickness are designated as $L, L_{T}, R$ and $t$, respectively.

\section{Imperfection Measurements}

Three-dimensional surveys of the inner and outer 
shell-wall surfaces of the specimens were made prior to testing the specimens to determine their initial geometric shell-wall imperfection shapes and shell-wall thickness distributions. Measurements were taken over a uniform grid with increments of 0.125 in. in the axial direction and $0.139 \mathrm{in}$. (approximately $1^{\circ} \mathrm{of}$ arc) in the circumferential direction over the exposed surfaces of the specimens. The inner surface measurement was used to determine the initial geometric shell-wall imperfection shape of a specimen, and the difference between the outer and inner surface measurements was used to determine the shell-wall thickness distribution. A contour plot of the nondimensionalized initial geometric shell-wall mid-surface imperfections $\bar{w}_{o}(x, \theta)$ for specimen $\mathrm{C} 3$ is shown in Fig. 2. The measured shell-wall imperfection $w_{o}$ is nondimensionalized by the average measured shell-wall thickness $t_{\text {ave }}=$ 0.0381 inches. These results indicate that the initial geometric shell-wall imperfection is periodic in the circumferential direction and has slight deviations in the axial direction. The amplitude of the imperfection varies from $+1.341 t_{\text {ave }}$ to $-1.535 t_{\text {ave }}$. A contour plot of the nondimensionalized shell-wall thickness variation $\bar{t}_{o}(x, \theta)$ for specimen C3 is shown in Fig. 3, where the measured thickness value $t_{o}$ is nondimensionalized by the average measured shell-wall thickness $t_{\text {ave }}$. These results indicate that the shell-wall thickness, and hence the laminate stiffnesses, varies significantly over a short distance. The thickness varies from 0.928 to 1.321 times $t_{\text {ave. }}$. Most of the thickness variation is attributed to local variations in the resin content of the laminate associated with the fabrication process. However, the darker angular pattern in the thickness distribution is attributed to small gaps between adjacent pieces of graphite-epoxy tape in some of the laminate plies that were generated during the lay-up and curing processes. Such a region is referred to herein as a lamina ply-gap or a ply-gap. In the particular case of shell $\mathrm{C} 3$, such a locally thin region in the shell wall consists of a 7-ply-thick laminate rather than the nominal 8-ply-thick laminate. For the case where one ply-gap intersects another plygap, the shell wall consists of a 6-ply-thick laminate. Moreover, these locally thin shell-wall regions have a significant shell-wall mid-surface eccentricity, and have reduced stiffnesses relative to the rest of the shell wall. Typically, a circumferential ply-gap is caused by a gap between two adjacent $90^{\circ}$ pieces of tape in the ply, a $45^{\circ}$ or helical ply-gap is caused by a gap between two adjacent $45^{\circ}$ pieces of tape, and an axial ply-gap is caused by a gap between two adjacent $0^{\circ}$ pieces of tape.
Magnified cross-sectional views of typical ply-gaps in a $-45^{\circ}$ outer ply of a specimen laminate and in a $90^{\circ}$ mid-surface ply are shown in Figs. $4 \mathrm{a}$ and $4 \mathrm{~b}$, respectively. The widths of the ply-gaps shown in Figs. 4a and $4 \mathrm{~b}$ are on the order of 0.02 in. or approximately equal to half the shell-wall thickness, and the ply-gap depth is approximately 0.005 in. or approximately equal to the nominal lamina ply thickness. In addition, there often exist regions adjacent to the ply-gaps in which a gradual thickness reduction of the lamina ply occurs as shown in Fig. 4a. These regions of lamina ply thickness reduction can extend an additional 0.02 to 0.04 in. from the edges of the ply-gap. Lamina ply-gaps with gap widths as large as 0.1 in. have been observed in some of the shell specimens. The lighter angular patterns in the thickness contour plot are caused by locally thickened regions of the outermost plies of the laminate that develop during the curing process to form outer shell-wall surface ridges. A magnified cross-sectional view of such a region is shown in Fig. 4c. Some of the shell-wall thickness features, such as lamina ply-gaps, are smaller than the imperfection-measurement grid spacing used in this study, and as a result, some of the smaller thickness variation features may not have been included in the measurements.

Measurements of the top and bottom loading surfaces of the specimens were made every degree around the circumference of the specimens to determine the variation in the shell-end or loading-surface geometry. Typical top and bottom shell-end or loading-surface geometry variations for specimen $\mathrm{C} 3$ are denoted by $\delta_{t o p}(\theta)$ and $\delta_{b o r}(\theta)$, respectively, and are shown in Fig. 5. The maximum amplitude of this shell-end or loading-surface variation is approximately 0.0015 inches, which is approximately $4 \%$ of $t_{\text {ave }}$ or $0.01 \%$ of the specimen length.

\section{Test Apparatus and Tests}

The specimens were instrumented with electrical resistance strain gages, and direct-current differential transducers (DCDT's) were used to measure displacements. Three non-collinear DCDT's were positioned at three corners of the upper loading platen of the test machine and used to measure the end-shortening displacement $\Delta$ and the rotations $\phi_{y}$ and $\phi_{z}$ of the loading platen as illustrated in Fig. 1. A typical set of measured loading platen rotations is shown in Fig. 6. These results indicate that significant upper platen rotations $\alpha$ cur from the onset of loading up to a load value of approximately $6,000 \mathrm{lbs}$. These rotations are attributed to an initial misalignment of the upper loading platen and the specimen. The rotations of the movable upper loading platen reach a steady state at a load value of 
$6,000 \mathrm{lbs}$., and the loading of the specimen, for the most part, continues without additional upper loading paten rotations from $6,000 \mathrm{lbs}$. up to the buckling or collapse load. During the collapse event, the upper loading platen undergoes an additional amount of rotation. The shadow moiré interferometry technique was used to observe the shell-wall prebuckling, buckling and postbuckling normal (perpendicular to the shell outer surface) deformation patterns. All data were recorded with a data acquisition system, and the moiré patterns were recorded photographically and on videotape.

The specimens were loaded in compression with a 300,000 -lb hydraulic universal-testing machine by applying an end-shortening displacement to the shell ends or loading surfaces of the specimens. To control the load introduction into the specimens, the upper loading platen was aligned with the loading surface of the specimen as well as possible before the test by adjusting leveling bolts in the corners of the upper loading platen until strains measured by selected strain gages on the specimens indicated a uniform axial strain distribution in the shell wall. The specimens were loaded until general instability or failure of the shells occurred.

\section{Finite-Element Models and Analyses}

\section{Nonlinear Analysis Procedure}

The shells considered in this study were analyzed with the STAGS (STructural Analysis of General Shells) nonlinear shell analysis code. ${ }^{13}$ STAGS is a finite-element code developed for the nonlinear static and dynamic analysis of general shells, and includes the effects of geometric and material nonlinearities in the analysis. The code uses both the modified and full Newton methods for its nonlinear solution algorithms, and accounts for large rotations in a shell by using a corotational algorithm at the element level. The Riks pseudo arc-length path-following method ${ }^{14}$ is used to continue a solution past the limit points of a nonlinear response. With this strategy, the incrementally applied loading parameter is replaced by an arc-length along the solution path, which is then used as the independent loading parameter. The arc-length increments are automatically adjusted by the program as a function of the solution behavior. The transient analysis option in STAGS uses proportional structural damping and an implicit numerical time-integration method developed by Park. ${ }^{15}$ Additional information on the transient analysis procedure can be found in Ref. 16.

The prebuckling, buckling and postbuckling responses of the shells were determined using the following analysis procedure. The prebuckling responses were determined using the geometrically nonlinear quasi-static analysis capability in STAGS. The Riks pseudo arc-length path-following method in STAGS was used to compute the initial shell response until just before buckling occurred. The unstable buckling response of the shell was predicted using the nonlinear transient analysis option of the code. The transient analysis was initiated from an unstable equilibrium state close to the limit point by incrementing the end displacement by a small amount. An initial time step of 1.0E-8 seconds was used in the analysis, and the time step was automatically adjusted by the program as a function of the solution behavior. The transient analysis was continued until the kinetic energy in the shell had dissipated to a negligible level, which indicated that the transient response had attenuated. Once the transient analysis had attenuated to a near-steady-state solution, the load relaxation option of the code was used to establish a static equilibrium state. Conventional linear bifurcation buckling analysis results were also determined with STAGS for comparison with the nonlinear response results.

\section{Finite-Element Models}

A typical finite-element model of a specimen is illustrated in Fig. 1. The standard 410 quadrilateral element from the STAGS element library was used in the models. The elements of the finite-element mesh are approximately 0.2 -in. by 0.2 -in. square. Each element has four integration points, which are distributed in such a way as to provide a modeling resolution of approximately 0.1 -in. by 0.1 -in square. This integrationpoint spacing is on the order of the measurement- point spacing used when measuring the initial geometric imperfections of the specimens. This highly refined mesh is necessary to model rapidly varying geometric and material parameters such as nonuniform shell-wall thicknesses and lamina stiffness properties. A typical finite-element model contained approximately 100,000 degrees of freedom.

Geometrically perfect and imperfect shells were analyzed in the present investigation. Nominal shell geometry, laminate thickness, lamina mechanical properties, and boundary conditions were used for the finiteelement models of the geometrically perfect shells. The nominal boundary conditions consist of setting the circumferential and normal displacements $v$ and $w$ equal to zero in the 1.0-in.-long potted boundary regions of the shell illustrated in Fig. 1, setting $u(L / 2, \theta)=0$, and applying a uniform end-shortening $u(-L / 2, \theta)=\Delta$.

The geometrically perfect finite-element models were modified to include the effects of the measured shell imperfections in order to simulate more closely the response of the specimens. These modeling modifications include the effects of the measured initial geometric shell-wall mid-surface imperfections, shell-wall 
thickness variations, local shell-wall lamina ply-gaps, thickness-adjusted lamina properties, elastic boundary conditions, shell-end geometric imperfections, and nonuniform end loads.

The initial geometric shell-wall mid-surface imperfection $w_{0}(x, \theta)$ is included in the finite-element models by introducing an initial normal perturbation to each node of the mesh by using a user-written subroutine with STAGS for that purpose. The user-written subroutine uses a linear interpolation algorithm that calculates the value of the imperfection for the coordinates of each finite-element node based on the measured shell-wall data.

The shell-wall thickness $t$, mid-surface eccentricity $e c z$, and lamina material properties $E_{l}, E_{2}, G_{12}$ and $v_{/ 2}$ are adjusted at each integration point of each element in the finite-element models. The shell-wall mid-surface eccentricity is calculated relative to the average shell-wall mid-surface as illustrated in Fig. 7; that is, ecz $(x, \theta)=-0.5\left(t_{\text {ave }}-t_{o}(x, \theta)\right)$. The lamina properties are adjusted by using the rule of mixtures. In the ruleof-mixtures calculations, it is assumed that any variation in the lamina ply thickness from the nominal thickness is due to a variation in resin volume only, and that the fiber volume remains constant for each ply. However, there are several assumptions and approximations related to modeling the geometry and stiffnesses of a lamina ply-gap detail that were used in the analysis. First, the finite-element models are limited to modeling the shell-wall thickness variation as discrete stepchanges, as illustrated in Fig. 7, and the resolution of the thickness variation is limited by the finite-element integration point spacing (i.e., 0.1 in.). Results from a study illustrating the potential effects of these modeling approximations and mesh refinement on the response of a shell with a ply-gap indicate that this modeling approach can affect the predicted response. It was found that these modeling approximations can lead to an artificial increase in the size of the measured thickness details, e.g., the width of a lamina ply-gap, resulting in misrepresentation of the local bending stiffnesses and the mid-surface eccentricity of the shell wall at that particular point. In addition, the mesh refinement and integration point spacing used in the present models tend to provide models that are overly stiff in bending by a small amount when they are used for modeling a plygap detail. These modeling approximations can have a direct influence on the local bending response of the shell and can result in as much as a $2 \%$ variation in the predicted buckling loads.

The second assumption is related to the modeling of the stiffnesses of the lamina ply-gap. Two modeling approaches were considered. One approach is to model each ply-gap with a local reduction in thickness, as measured, including the local eccentricity $e c z$, and reducing the appropriate number of lamina plies in the shell-wall laminate model, hence, reducing the local stiffnesses associated with the local ply-gap detail. The second approach is to model the ply-gap with the local as-measured reduced shell-wall thickness and the corresponding local mid-surface eccentricity, but assuming that the local thickness reduction is due to a reduction in the resin volume only and, consequently, keeping the fiber volume constant. This approach neglects the local stiffness reduction associated with the ply-gap details. The former modeling approach is very time consuming to implement since it requires the manual definition of each shell-wall laminate associated with each specific ply-gap in the finite-element model. The latter modeling approach is much easier to implement in the model by using a user-written subroutine compatible with the STAGS finite-element analysis code. As a result, the latter modeling approach was used in the present study. Results from a study of this modeling assumption indicate that neglecting the local stiffness reduction associated with a ply-gap would cause only slight differences in the magnitude of the local bending response and no more than a $2 \%$ variation in the predicted buckling load for a compression-loaded shell. In addition, the results of this modeling study indicated that the local shell-wall mid-surface eccentricity is the most important feature of the ply-gap detail for these stability critical problems, and this eccentricity was included in the models for the results presented herein.

To provide a better simulation of the constraints provided by the potting material at the ends of the specimens, effective axial and radial potting-support stiffnesses were determined for each shell specimen using a two-dimensional generalized plane-strain finite-element analysis of the potting-material-shell-wall detail shown in Fig. 8a. Material properties of the potting compound were characterized by Iosipescu tests of the potting material reported by Weiland et al. ${ }^{17}$ The nominal properties of the potting material are as follows: Young's modulus $E=1.15 \mathrm{Msi}$, shear modulus $G_{12}=$ $0.36 \mathrm{Msi}$, and Poisson's ratio $v=0.59$. The effective laminate axial stiffness $E_{x}$ was used in the shell-wall models which include $E_{x}$ equal to $11.83,3.60,8.07$, and $16.14 \mathrm{Msi}$, for shells $\mathrm{C} 1, \mathrm{C} 2, \mathrm{C} 3$ and $\mathrm{C} 4$, respectively. To determine the effective axial and radial potting-support stiffnesses, the following numerical experiments were conducted. In the first numerical experiment, a uniform axial displacement $\Delta$ was applied to an unpotted end of the shell model, and the resulting predicted axial strain response, shown in Fig. 8b, was used to calculate an effective axial stiffness $K_{A}$ for the 
portions of the shell wall supported by the potting material. The results indicate that the axial strain response of the shell wall supported by the potting material is a function of shell-wall laminate modulus. In particular, the decay length of the strain response varies inversely with the axial laminate stiffness. The predicted effective axial potted-shell stiffnesses are $26.55,6.73,26.26$ and $32.37 \mathrm{Msi}$ for shells $\mathrm{C1}, \mathrm{C} 2, \mathrm{C} 3$ and C4, respectively. In the second numerical experiment, a unit concentrated force $F_{z}$ was applied to the shell model, and the resulting $w$ (radial or normal) displacement and nodal force at the junction of the shell wall and the potting material was used to calculate an effective radial support stiffness $K_{R}$. The effective radial potting-support stiffness was predicted to be approximately equal to $1.0 \mathrm{E} 5 \mathrm{lbf} / \mathrm{in}$.

Nonuniform end loading of a specimen is attributed to initial specimen-end or loading-surface imperfections and to upper loading-platen rotations that are measured during the experiment. First, the measured upper and lower specimen-end or loading-surface imperfections $\delta_{t o p}(\theta)$ and $\delta_{b o r}(\theta)$, respectively, were included in the finite-element model by introducing an initial in-plane axial perturbation to the nodes at the loaded ends of the shell. Then, the compression load was applied to the shell in two parts. The nonuniform specimen-end imperfections, $-\delta_{\text {top }}(\theta)$ and

$\delta_{b o t}(\theta)$, were applied as displacements to the upper and lower ends of the shell, respectively, at the beginning of the analysis to simulate a full contact condition between the shell ends and the loading platens. Then, the experimentally measured end-shortening displacement $\Delta$ and upper loading-platen rotations $\phi_{y}$ and $\phi_{z}$ were applied to the upper shell end or loading surface while holding the lower loading surface fixed as illustrated in Fig. 1; that is, $u(-L / 2, \theta)=\Delta+R \cos \phi_{y} \cos \theta+R \cos$ $\phi_{z} \sin \theta-\delta_{\text {lop }}(\theta)$ and $u(L / 2, \theta)=-\delta_{b o t}(\theta)$.

\section{Results and Discussion}

Analytically predicted and experimentally measured results for the four compression-loaded graphiteepoxy cylindrical shells considered in this study are presented in this section. The shell-wall laminates of the four shells include two different orthotropic laminates and two different quasi-isotropic laminates. The two 8-ply orthotropic shells, $\mathrm{Cl}$ and $\mathrm{C} 2$, and the 8-ply quasi-isotropic shell, C3, have shell-radius-to-thickness ratios equal to 200 . The 16-ply quasi-isotropic shell, $\mathrm{C} 4$, has a shell-radius-to-thickness ratio equal to 100 . The predicted results were obtained from finite-element models of geometrically perfect shells and shells that include initial geometric shell-wall mid-surface imperfections, shell-wall thickness variations and thickness- adjusted lamina properties, local shell-wall lamina plygaps, elastic boundary support conditions, and nonuniform loading effects. These results are presented to illustrate the overall behavior of compression-loaded graphite-epoxy shells and the effects of imperfections on their response. First, results illustrating the effects of orthotropy on the nonlinear response of the 8-ply shells are presented. Next, results illustrating the effects of initial shell geometric and loading imperfections on the buckling response of the 8-ply shells are presented. Then, results illustrating the effects of manufacturing defects in the form of lamina ply-gaps on the nonlinear response of the 8-ply quasi-isotropic shell are presented. Results illustrating the effects of elastic boundary support conditions on the displacement and strain response of selected shells are also presented. In addition, results that demonstrate the effects of uncertainties or variations in selected parameters on shell response are presented. These uncertainties include uncertainties in the lamina fiber volume fraction, applied loading conditions, and imperfection measurements. Finally, comparisons between selected analytically predicted results and experimentally measured results for all four shells are presented. The results include predicted and measured load-endshortening response curves, predicted and measured load-strain response curves, predicted prebuckling, buckling and postbuckling deformation response patterns, and predicted axial and circumferential stress resultant patterns. In addition, the results of a local twodimensional stress analysis which identify severe local stress gradients in the shell wall of specimen $\mathrm{C} 4$, which could have caused the specimen to fail before it buckled, are presented. The four composite shells considered in this study are interchangeably referred to herein as shells or specimens $\mathrm{C} 1, \mathrm{C} 2, \mathrm{C} 3$ and $\mathrm{C} 4$.

\section{Effects of Orthotropy and Anisotropy on the Nonlinear Response of Geometrically Perfect Shells}

Results from the nonlinear analyses of selected geometrically perfect shells are presented in this section. The shells analyzed are based on shells C1, C2 and $\mathrm{C} 3$ which have orthotropic shell-wall laminates $\left[\mp 45 / 0_{2}\right]_{\mathrm{s}}$ and $\left[\mp 45 / 90_{2}\right]_{\mathrm{s}}$, and a quasi-isotropic shellwall laminate $[\mp 45 / 0 / 90]_{s}$, respectively. First, the load-end-shortening response curves for the shells are compared. Next, results illustrating a typical nonlinear transient collapse response for the quasi-isotropic shell C3 are presented in detail. Finally, results illustrating the effects of orthotropy and anisotropy on the nonlinear response are presented. Values of the axial load $P$ and the end-shortening $\Delta$ presented in this section are normalized by the predicted linear bifurcation buckling 
load for the quasi-isotropic shell C3, $P_{\text {hif }}^{\text {quasi }}=42,590$ Ibs., and the nominal shell-wall thickness, $t_{n o m}=0.04$ in., respectively.

The predicted load-end-shortening responses are compared for the three shells in Fig. 9a. The prebuckling load-end-shortening responses are linear up to the general instability point indicated in the figure for each shell. The prebuckling slopes of each response curve vary according to the individual laminate in-plane axial stiffnesses. General instability occurs at normalized axial load values of $0.707,0.868$ and 0.979 , for shells $C 1$, $\mathrm{C} 2$ and $\mathrm{C} 3$, respectively. The general instability response is followed by a sudden reduction in the axial load supported by the shells which is associated with the transient collapse response of the shells. The corresponding load-time histories of the transient collapse response of the shells are shown in Fig. 9b. The initial portion of a typical load-time history curve, e.g., the load-time history curve for shell $\mathrm{C} 1$, is associated with the transition from a state just before buckling occurs (time $=0.0$ ) to the state associated with the general instability point indicated in the figure. This initial portion of the load-time history curve only occurs in the analysis of the geometrically perfect shells considered in the present study, and is attributed to the uniformly symmetric character of the initial response. The nonlinear solution algorithm that predicts the structural response of the shell needs a small perturbation in the response to be able to change from the axisymmetric prebuckling response state to an asymmetric general instability and transient collapse response state in the analysis. Since there are no such geometric or loading perturbations in the model for the geometrically perfect shells, it is suspected that small numerical variations in the transient solution accumulate and provide a small perturbation to the equilibrium state of the shell that causes the general instability and transient collapse response to occur. Following the initiation of the general instability response, a typical load-time history curve exhibits a sudden reduction in the axial load supported by the shell which is associated with the transient collapse of the shell. The value of the axial load continues to decrease until the axial load attenuates to a steadystate value. The kinetic energy in the shell dissipates over time and the shell reaches a stable postbuckling equilibrium state after approximately 0.035 to $0.04 \mathrm{sec}-$ onds have elapsed. Normalized post-collapse postbuckling load values equal to $0.403,0.218$ and 0.286 are obtained for shells $\mathrm{C} 1, \mathrm{C} 2$ and $\mathrm{C} 3$, respectively. The results indicate that laminate orthotropy can have a significant effect on the load-end-shortening response of the shells. In particular, while shell $\mathrm{C} 1$ has the lowest general instability load value of all three shells, it also has the highest postbuckling load value of all three shells.

The transient deformation responses for selected time steps during the transient collapse of quasi-isotropic shell $\mathrm{C} 3$ are presented in Figs. 10a through 10f. The deformation responses shown in the figures have been scaled for clarity. The shell-wall normal deformations at the general instability point shown in Fig. $9(0.0125$ seconds into the predicted collapse response) change in a short period of time from the uniform axisymmetric prebucking deformation pattern shown in Fig. 10a to the asymmetric transient unstable deformation pattern shown in Fig 10b, at which time the shell begins to collapse. The magnitudes of the shell-wall normal displacements vary between \pm 0.5 times the shell-wall thickness. The general instability response of the shell is caused by the nonlinear coupling of the localized destabilizing compressive axial and circumferential stresses in the shell indicated in Figs. $11 \mathrm{a}$ and $11 \mathrm{~b}$ and the normal deformations that occur in the bending boundary layer near the ends of the shell. The initial buckling deformation pattern indicated in Fig. 10b is characterized by localized ellipse-like buckles in the bending boundary layer near the ends of the shell. The skewing of the deformation response is attributed to the presence of the small anisotropic bending-twisting coupling stiffness terms of the laminate. After $0.013 \mathrm{sec}$ onds have elapsed, the normalized axial load has decreased from 0.983 to 0.479 , and the deformation response in the shell has evolved to include additional rows of small ellipse-like buckles as indicated in Fig. 10c. The magnitudes of the shell-wall normal displacements vary from +2 to -4 times the shell-wall thickness. After 0.0134 seconds have elapsed, the normalized axial load has decreased to 0.465 , and the local buckles in the deformation pattern have rapidly increased in number to include 24 circumferential half-waves and four axial half-waves as indicated in Fig. 10d. After 0.0152 seconds have elapsed, the normalized axial load has decreased further to 0.426 , at which time the small buckles in the shell wall begin to coalesce into larger buckles with displacement magnitudes that vary from +3 to -7 times the shell-wall thickness as indicated in Fig. 10e. After approximately 0.04 seconds have elapsed, the kinetic energy in the shell has dissipated to a negligible level, and the shell has deformed into a stable postbuckling mode-shape that consists of 16 circumferential half-waves and two axial half-waves, as indicated in Fig. 10f.

The transient deformation responses for selected time steps during the transient collapse of axially stiff orthotropic shell $\mathrm{Cl}$ are presented in Figs. 12a through 12c. The over-all load-time history character of the transient collapse response of shell $\mathrm{Cl}$ is similar to that 
of the response exhibited by shell $\mathrm{C} 3$, but there are some differences in the predicted deformation palterns and the collapse initiation events exhibited by shells $\mathrm{C} 1$ and $\mathrm{C}$. The deformation pattern of shell $\mathrm{Cl}$ just before buckling occurs is characterized by a uniform short wave-length axisymmetric response along the entire length of the shell as shown in Fig. 12a. These results indicate that the bending boundary layer response does not attenuate for the laminate of shell $\mathrm{Cl}$, which is in contrast to the attenuated bending boundary layer response exhibited by shell $\mathrm{C} 3$ as shown in Fig 10a. At the general instability point shown in Fig. 9 (after 0.0167 seconds have elapsed in the transient collapse response), a circumferential wave pattern has developed into the shell-wall normal deformations for shell $\mathrm{Cl}$ as indicated in Fig. 12b, which is in contrast to the localized response that occurs in the bending boundary layer near the ends of shell C3 as shown in Fig. 10b. The global collapse of shell $\mathrm{Cl}$ initiates in the interior of the shell instead of initiating in the bending boundary layer as it did for shell $\mathrm{C} 3$. In addition, shell $\mathrm{Cl}$ does not exhibit the pronounced skewing of the deformation response that was indicated for shell $\mathrm{C} 3$, which suggests that this shell is less sensitive to the effects of the small anisotropic bending-twisting coupling stiffness terms of the laminate. After 0.04 seconds have elapsed in the transient collapse response, shell $\mathrm{Cl}$ exhibits a postbuckling deformation pattern that is characterized by 16 circumferential half-waves and one axial halfwave as indicated in Fig 12c. This pattern is in contrast to the pattern of shell $\mathrm{C} 3$ which has a postbuckling deformation pattern with 16 circumferential half-waves and two axial half-waves.

The transient deformation responses for selected time steps during the transient collapse of circumferentially stiff orthotropic shell $\mathrm{C} 2$ are presented in Figs. 13a through 13c. The over-all character of the transient collapse response of shell $\mathrm{C} 2$ is similar to the response exhibited by shell $\mathrm{C} 3$, however, there are some differences in the resulting deformation patterns exhibited by shell $\mathrm{C} 2$. The deformation pattern for shell $\mathrm{C} 2$ just before buckling occurs is characterized by a uniform short-wave-length axisymmetric bending boundary layer response that attenuates rapidly into the interior of the shell as shown in Fig. 13a. At the general instability point shown in Fig. 9 (after 0.0217 seconds have elapsed in the transient collapse response), the shell exhibits a deformation pattern that is characterized by one axial half-wave and 22 circumferential half-waves as indicated in Fig 13b. This pattern is in contrast to the short-wave-length responses exhibited by shells $\mathrm{Cl}$ and C3 shown in Figs. 12b, and 10b, respectively. In addition, the shell-wall deformations exhibit a significant amount of skewing that is attributed to the presence of the small anisotropic bending-twisting coupling stiffness terms of the laminate. After 0.037 seconds have elapsed in the transient collapse response, shell $\mathrm{C} 2 \mathrm{ex}$ hibits a stable postbuckling deformation pattern that is characterized by 14 circumferential half-waves and two axial half-wave as indicated in Fig 13c. This pattern is in contrast to the pattern for shell $\mathrm{C} 3$ which exhibits a postbuckling deformation pattern with 16 circumferential half-waves and two axial half-waves.

The previous results indicate that laminate orthotropy can have an effect on the nonlinear response of compression-loaded composite shells. In particular, the results indicate that the bending boundary layer attenuation response can be significantly affected by the orthotropy of the laminate. The effects of laminate orthotropy on the displacement profiles just before buckling occurs are indicated in Fig. 14 for the three shells. The dashed, dash-dot, and solid lines represent displacement profiles for shells $\mathrm{C} 1, \mathrm{C} 2$ and $\mathrm{C} 3$, respectively. These results indicate that the axially stiff orthotropic shell $\mathrm{Cl}$ exhibits large-amplitude short-wavelength bending deformations just before buckling occurs. In addition, the bending response decays only slightly over the length of this shell. In contrast, shells $\mathrm{C} 2$ and $\mathrm{C} 3$ exhibit short-wave-length bending boundary layer responses that attenuate into the interior of the shell. These results also indicate that the laminate orthotropy can have a significant effect on the magnitude of the maximum displacements in the bending boundary layer near the ends of the shell. The displacements with the largest magnitude are exhibited by shell $\mathrm{Cl}$ which has a maximum displacement magnitude of approximately $0.34 w / t_{\text {nom }}$ followed by shells $\mathrm{C} 2$ and C3 which have maximum displacement magnitudes equal to 0.29 and $0.24 w / t_{n o m}$, respectively. Additional results are presented in Fig. 15 that indicate the effects of shell length on the bending boundary layer attenuation characteristics of shell $\mathrm{Cl}$. The solid, dashed, and dash-dot lines in the figure represent displacement profiles for shell length-to-radius ratios $L R$ equal to 2,3 and 4 , respectively. These results indicate that the bending boundary layer response for the shell $\mathrm{Cl}$ laminate attenuates for the longer shells, but not for the shorter shell. In addition, the results indicate that the magnitude of the large-amplitude displacements near the ends of the shell decreases as the length of the shell increases. These results suggest that the non-attenuating bending boundary layers in the shorter shell interact to cause displacements with larger magnitudes in this shell. These results also suggest that the effects of the attenuation length of the bending boundary layer should be considered in studies of the nonlinear stability response and failure characteristics of orthotropic shells 
and in the design of these shells.

Effects of Initial Imperfections on the Buckling Response

Results from a parametric study that illustrate the effects of initial imperfections on the nonlinear response of compression-loaded composite shells are presented in this section. The imperfections studied include traditional initial geometric shell-wall mid-surface imperfections and other nontraditional shell-wall imperfections and nonuniform load distribution effects. The nontraditional shell-wall imperfections include the effects of measured shell-wall thickness variations and corresponding thickness adjusted lamina properties. The nonuniform load distribution conditions include the effects of initial shell-end imperfections and measured applied load variations. Imperfection-amplitude scaling factors or coefficients were introduced into the models to allow for independent variations in the imperfection amplitudes during the study. Imperfectionamplitude scaling factors for the shell-wall mid-surface imperfection, shell-wall thickness imperfection, and load distribution imperfection are denoted by $W, T$ and $D$, respectively. Imperfection-amplitude scaling factor values equal to $0.0,0.5$ and 1.0 were considered in the present study. An imperfection-amplitude scaling factor equal to 1.0 indicates that the actual amplitude of the measured imperfection was used in the analysis, and a value equal to 0.0 indicates that the imperfection was not included in the analysis. An imperfection-amplitude scaling factor equal to 0.5 indicates that half of the measured imperfection amplitude was included in the analysis. First, typical results from the parametric study illustrating the effects of measured imperfections on the buckling load of quasi-isotropic shell $\mathrm{C} 3$ are presented. Then, typical results illustrating the effects of measured imperfections on the nonlinear collapse response of shell C3 are presented.

Results illustrating the effects of measured imperfections on the predicted buckling load of quasi-isotropic shell C3 are shown in Fig. 16 where the buckling load $P_{c r}$ is normalized by the linear bifurcation buckling load $P_{b i f}$. The open square symbols in the figure represent the buckling loads of the shell for different values of the parameters considered. The solid and dashed lines represent thickness imperfection-amplitude scaling factors of $T$ equal to 0.0 and 1.0 , respectively. The results indicate that the measured imperfections can have a significant effect on the buckling load of the shell with the normalized buckling loads ranging from 0.947 to 0.842 . These results indicate that the magnitude of the buckling load is most sensitive to the traditional shell-wall mid-surface geometric imperfection which can account for as much as a $7 \%$ reduction in the buckling load of the shell. The results also indicate that the shell-wall thickness variation can account for a $3-4 \%$ reduction in the bucking load. In addition, the results indicate that the nonuniform load distribution imperfection can cause a reduction in the buckling load of approximately $3-4 \%$ for small values of the geometric shell-wall mid-surface imperfection amplitude. However, as the amplitude of the geometric shell-wall mid-surface imperfection increases, the nonuniform load distribution affects the amount of the decrease in the buckling load differently. This result indicates that there exists a complex nonlinear coupling behavior between the shell-wall mid-surface imperfection and the nonuniform load distribution for this shell. In particular, the character of the nonuniform loading is such that, when it couples with the geometric shell-wall mid-surface imperfections, the shell exhibits a redistribution of the internal loads to regions of the shell that have higher geometric stiffness values which, in turn, retards to onset of buckling in the shell. Results for $T=0.5$ exhibit similar trends, and the buckling load values are 1 to $2 \%$ lower than those for $T=$ 0.0 .

Similar results for orthotropic shells $\mathrm{C} 1$ and $\mathrm{C} 2$ indicate that these shells are apparently less sensitive to the imperfections considered in the parametric study since they exhibit reductions in buckling load values of $4.1 \%$ and $4.7 \%$, respectively, which is in contrast to the $11 \%$ reduction exhibited by shell $C 3$. These results also indicate that, while the buckling loads are most sensitive to variations in the traditional shell-wall geometric imperfection amplitude, this geometric imperfection results in only a 1.7 and a $4.0 \%$ reduction in the buckling loads of shells $\mathrm{C} 1$ and $\mathrm{C} 2$, respectively, which is in contrast to the $7 \%$ reduction exhibited by shell $\mathrm{C} 3$. In addition, the shell-wall thickness imperfection and nonuniform load distribution imperfection both appear to cause no more than a $1 \%$ reduction in the buckling loads of these shells, which is in contrast to the $3-4 \%$ reduction exhibited by shell C3.

Typical analytically predicted transient response deformations that occur during the collapse of shell C3 with imperfections included in the analysis are shown in Figs. 17a through 17d. The imperfection-amplitude scaling factors for these results are $W=1.0, T=1.0$, and $D=1.0$. Just before buckling occurs, the shell wall deformations are characterized by several localized ellipse-like deformation patterns as indicated in Fig. 17a. The localization in the deformation pattern is caused by the combination of a local geometric shell-wall imperfection that is in the form of a significant variation in the shell-wall mid-surface geometry, and the intersection of a helical ply-gap and a circumferentially aligned ply- 
gap in the shell at $r / L_{T}=0.25$ and $\theta=210^{\circ}$. The localized deformations occur in regions with destabilizing compressive axial and circumferential stresses as indicated in Fig. 18. After approximately 0.00143 seconds have elapsed in the transient response, a single ellipselike buckle has grown in amplitude and couples with the destabilizing stresses in the shell wall to cause the general instability and collapse of the shell. After approximately 0.00238 seconds have elapsed in the transient response, additional local buckles have formed around the circumference and along the length of the shell as indicated in Fig. 17c. As the buckling process continues, the deformation pattern in the shell wall continues to evolve, and the ellipse-like buckles in the shell begin to coalesce into larger diamond-shaped buckles. After approximately 0.02 seconds have elapsed in the transient response, the kinetic energy in the shell has dissipated to a negligible level, and the shell has deformed into a stable postbuckling mode-shape as indicated in Fig. $17 \mathrm{~d}$. These results indicate that the collapse response of the imperfect shell is initiated by a localized response which leads to the over-all collapse of the shell. These results are different from the collapse response results exhibited by the corresponding geometrically perfect shell which are shown in Figs. 10a-10f. The transient collapse response occurs after 0.00143 seconds have elapsed in the transient analysis in the shell with imperfections, and this collapse response occurs much earlier in the load-response history of the shell than for the corresponding geometrically perfect shell collapse response. This earlier collapse time is attributed to the localized prebuckling shell-wall deformations and destabilizing in-plane compressive stresses in the shell, which result in a rapid transition from the stable prebuckling state to the unstable transient collapse response. Results for orthotropic shells $\mathrm{C} 1$ and $\mathrm{C} 2$ indicate similar response characteristics associated with the transient collapse response of these shells.

\section{Effects of Lamina Ply-Gap Fabrication Defects}

Selected results illustrating the effects of lamina ply-gaps on the response of quasi-isotropic shell $\mathrm{C} 3$ are presented in this section. First, results illustrating the effects of lamina ply-gap orientation, ply-gap width, and ply-gap depth on the buckling load of this shell are presented. Ply-gap orientations of $-45^{\circ},+45^{\circ}, 0^{\circ}$ and $90^{\circ}$; ply-gap widths equal to 0.1 in. and 0.2 in.; and plygap depths equal to $0.005 \mathrm{in}$. and 0.0025 in. are considered. Then, typical results illustrating the effects of a helical ply-gap on the transient collapse response of the shell are presented.

Results illustrating the effects of ply-gap orienta- tion, width, and depth on the buckling load of shell $C 3$ are shown in Fig. 19 where the buckling load $P_{c r}$ is normalized by the linear bifurcation buckling load $P_{b i f}$. The open square symbols represent calculated normalized buckling loads. The solid and dashed lines represent result trends for ply-gap depths of 0.005 in. and 0.0025 in., respectively. The results indicate that the buckling load of the quasi-isotropic shell can be affected by certain types of lamina ply-gaps. In general, as the ply-gap width and ply-gap depth increase, the buckling load decreases. The results also indicate that the buckling load is sensitive to the orientation of the plygap. These results indicate that shells with $90^{\circ}$ ply-gaps exhibit the most significant reductions in buckling load followed by shells with $-45^{\circ},+45^{\circ}$ and $0^{\circ}$ ply-gaps. The results also indicate that shells with $-45^{\circ}$ ply-gaps exhibit lower buckling loads than shells with $+45^{\circ}$ plygaps. These results suggest that there is a nonlinear coupling response that is affected by the laminate anisotropy and the local structural response associated with the lamina ply-gap. In addition, the results indicate that the buckling load of the shell is not sensitive to the effects of a $0^{\circ}$ ply-gap. The benign effect of the $0^{\circ}$ ply-gap suggests that the local bending deformations and destabilizing in-plane stresses near the ply-gap for this shell do not significantly affect the buckling of the shell.

Transient deformation response results for selected time steps during the transient collapse of quasi-isotropic shell $\mathrm{C} 3$ with a $-45^{\circ}$ lamina ply-gap are presented in Fig. 20a through 20d. Just before buckling occurs, the shell-wall deformations are characterized by a combination of a large local inward deformation pattern aligned with the ply-gap and a small ellipse-shaped deformation pattern located at the intersection of the bending boundary layer deformations near the end of the shell and the ply-gap as shown in Fig. 20a. The destabilizing in-plane axial and circumferential stresses shown in Fig. $21 \mathrm{a}$ and $21 \mathrm{~b}$, respectively, couple with the normal shell-wall deformations to cause the general instability and collapse of the shell to occur. After approximately 0.0012 seconds have elapsed in the transient response, additional ellipse-like or diamondshaped buckle patterns have formed in the shell in the vicinity of the ply-gap as indicated in Fig. 20b. After approximately 0.0029 seconds have elapsed in the transient response, additional local buckle patterns have formed around the circumference and along the length of the shell as indicated in Fig. 20c. As the buckling process continues, the deformation pattern in the shellwall continues to evolve, and the ellipse-like buckle patterns in the shell begin to coalesce into larger dia- 
mond-shaped buckle patterns. After approximately 0.02 seconds have elapsed in the transient response, the kinetic energy in the shell has dissipated to a negligible level, and the shell has deformed into a stable postbuckling mode-shape as indicated in Fig. 20d. Similar results for orthotropic shells $\mathrm{Cl}$ and $\mathrm{C} 2$ indicate that the nonlinear response and buckling loads of these orthotropic shells are only slightly affected by the lamina ply-gap features. For these orthotropic shells, the plygaps cause a maximum reduction in the predicted buckling loads of approximately $1-2 \%$. This benign effect of the ply-gaps on the buckling loads of these orthotropic shells suggests that the local bending deformations and destabilizing in-plane stresses associated with the plygaps do not significantly affect the buckling loads of these shells.

\section{Effects of Elastic Boundary Conditions}

The potting material applied to the ends of the shell specimens is intended to prevent premature damage to the ends of the shell during testing. In practice, it is virtually impossible to achieve perfect rigidly clamped supports in a test. It has been shown by Fuchs et al. ${ }^{18}$ that the displacement and strain responses in cylindrical shells subjected to an applied bending load can be sensitive to the flexibility of the specimen support conditions. To determine if the flexibility of the end supports may affect the response of compression-loaded shells, an analytical study was conducted to assess the effects of axial and radial potting-support stiffnesses on the response of the shells considered in the present study. Preliminary results indicate that the axial potting-support stiffness primarily affects the effective axial stiffness of the shell and has no noticeable effect on the displacement and strain response of the shell. However, the results also indicate that the radial potting-support stiffnesses can have a significant effect on the displacement and strain response of the shells, and some of these results are presented herein.

Selected results from a parametric study are presented to illustrate the effects of elastic radial constraints on the displacement and strain response of selected geometrically perfect and imperfect shells. Elastic radial stiffnesses $K_{R}$ equal to $1.0 \mathrm{E} 3,1.0 \mathrm{E} 5$ and $1.0 \mathrm{E} 7 \mathrm{lbf} / \mathrm{in}$. were considered in the study. A value of the elastic radial stiffness $K_{R}=1.0 \mathrm{E} 5 \mathrm{lbf} / \mathrm{in}$. corresponds to the effective radial potting-support stiffness calculated from the potting model illustrated in Fig. 8, and a value of $K_{R}=1.0 \mathrm{E} 7 \mathrm{lbf} / \mathrm{in}$. corresponds to a clamped condition. Results illustrating the effects of elastic radial potting-support conditions on the prebuckling displacements and strain responses of geometrically perfect and imperfect orthotropic shell $\mathrm{C} 2$ are presented subsequently.

Results illustrating the effects of elastic radial potting-support conditions on the typical prebuckling normal displacements of the geometrically perfect and imperfect orthotropic shell $\mathrm{C} 2$ are presented in Figs. $22 \mathrm{a}$ and $22 \mathrm{~b}$, respectively. The normal displacement $w$ and the axial coordinate $x$ are normalized with respect to the nominal shell-wall thickness $t_{\text {nom }}$ and the shell test-section length $L_{T}$, respectively. The solid, dashed, and dot-dashed lines in the figures represent results for shells with elastic radial potting-support stiffnesses $K_{R}$ equal to $1.0 \mathrm{E} 7,1.0 \mathrm{E} 5$ and $1.0 \mathrm{E} 3 \mathrm{lb} /$ in., respectively. The results indicate that the variation in the radial boundary stiffness can have a measurable effect on the magnitude of the displacements for the geometrically perfect shell as shown in Fig. 22a. These results indicate that radial boundary stiffnesses equal to $1.0 \mathrm{E} 5$ and $1.0 \mathrm{E} 7 \mathrm{lbf} / \mathrm{in}$. restrain the radial displacements at the boundaries of the shell. However, when the elastic radial boundary stiffness is reduced to $1.0 \mathrm{E} 3 \mathrm{lbf} / \mathrm{in}$., the boundaries of the shell have an outward radial displacement of approximately $0.06 \mathrm{w} / \mathrm{t}_{\text {nom }}$ and a reduction in the maximum displacements in the bending boundary layer of approximately $0.02 \mathrm{w} / \mathrm{t}_{\mathrm{nom}}$. In addition, the shell exhibits a slight axial shift in the bending boundary layer response near the ends of the shell. These results indicate that the variation in radial boundary stiffness can have a significant effect on the displacement response of a shell with imperfections as shown in Fig. 22b. The results indicate that there can be a 20 to $25 \%$ variation in the local displacement response at several locations along the length of the shell for the different boundary conditions. Other related results indicate that these variations in the radial boundary stiffness and variations in the prebuckling displacement response can cause significant changes in the character of the collapse and post-collapse deformation responses of the shells.

Results illustrating the effects of elastic radial support conditions on the prebuckling axial strain response of the geometrically perfect and imperfect orthotropic shell $\mathrm{Cl}$ are presented in Figs. 23a and 23b, respectively. The axial strains $\varepsilon_{x}$ and the axial coordinate $x$ are normalized by the axial strain at buckling $\varepsilon_{c r}$ and the shell test-section length $L_{T}$, respectively. These results indicate that variations in the elastic radial support conditions can have an influence on the strain response in the geometrically perfect and imperfect shells. The results indicate that the strain gradients near the ends of the shell shift in the axial direction, which cause a local variation in the strain values of as much as $15 \%$ and a reduction in the maximum strain of approx- 
imately $5 \%$. In addition, the results indicate that variations in the elastic radial support condition can have a similar effect on the strain response of an imperfect shell as shown in Fig. 23b. These results indicate that elastic radial support stiffnesses of $1.0 \mathrm{E} 3$ and $1.0 \mathrm{E} 5 \mathrm{lbf} /$ in. can cause a local variation in strain values of as much as $17 \%$ near the boundary of the shell. These results also suggest that including the effects of elastic radial support conditions in the analysis may be important when interpreting experimentally measured strain gage data for strain gages located near the ends of a specimen.

\section{Effects of Uncertainties or Variations in Selected Speci-} men Parameters

Results from a numerical study of the effects of uncertainties or variations of selected specimen parameters on the response of the imperfect quasi-isotropic shell C3 are presented in this section. The parametric uncertainties considered include uncertainties in the imperfection measurements, the lamina fiber volume fraction, and the applied load distribution. The imperfection measurement uncertainties are attributed to the accuracy tolerances of the coordinate measurement device used to measure the initial geometry of the shell. The shell-wall imperfection measurement values and the shell-end-shape imperfection measurement values used in the present study are accurate to within \pm 0.0006 inches, or $\pm 0.75 \mathrm{E}-4 R$ and $\pm 1.5 \mathrm{E}-4 L$. The shell-wall thickness values are accurate to within \pm 0.0012 inches, or $\pm 0.03 t_{\text {nom }}$. The lamina fiber volume fraction is specified by the manufacturer of the material to be equal to $0.65 \pm 0.03$ for the 0.005 -in.-thick graphite-epoxy preimpregnated tape material used to fabricate the test specimens. Applied load distribution uncertainties are measured indirectly by monitoring the measured and predicted axial strains at selected points near the top and bottom loading surfaces of the shell. A correction to the applied displacements can be determined from the differences in the measured and predicted strains as follows. A user-written program external to the STAGS code was used to analyze the differences in the measured and predicted strains for a specified applied load value. This program used an iterative predictor-corrector method to determine a correction to the applied shell-end displacements. A new finite-element analysis was conducted with this displacement correction included in the model. This process was repeated iteratively until the difference in the measured and predicted strains reached a predetermined tolerance. A typical predicted shell-end displacement correction for shell C3 is shown in Fig. 24. The solid and dashed lines represent the predicted top and bottom corrections to the displacements applied to the shell, respectively. The results indicate that significant corrections to the applied end displacements are predicted for this shell, and the magnitude of the corrections is on the order of the initial shell-end imperfections shown in Fig. 5. The majority of this correction is attributed to differences in the assumed orientation of the upper loading platen with respect to the shell specimen and its actual orientation prior to applying the load to the specimen.

Results illustrating the effects of uncertainties in several specimen parameters on the buckling load of the imperfect quasi-isotropic shell C3 are shown in Fig. 25. These parametric uncertainties include variations in the lamina fiber volume fraction, the measured shell-wall thickness imperfection, and the applied load distribution. The open square symbols in the figure represent calculated normalized buckling loads, where the buckling load $P_{c r}$ is normalized by the linear bifurcation buckling load $P_{b i f}$. The solid, dashed, and dot-dashed lines in the figure represent trends in the results for shells with thickness imperfection measurement variations of $-3 \%, 0 \%$, and $+3 \%$, respectively. These results indicate that the normalized buckling loads range from 0.83 to 1.0 , and that variations in these parameters can have a significant effect on the buckling load of the shell. In particular, the results indicate that a variation in the shell wall thickness of $\pm 3 \%$ can result in a $6 \%$ variation in the buckling load of the shell. Similarly, results indicate that a $\pm 3 \%$ variation in the fiber volume fraction can result in an $8 \%$ variation in the buckling load. In addition, the buckling load is decreased by up to $2 \%$ when the magnitude of the applied load distribution correction amplitude scaling factor is increased from 0.0 to 1.0. Results for shells $\mathrm{C} 1$ and $\mathrm{C} 2$ indicate similar tends. However, these results suggest that the orthotropic shells are less sensitive to predicted applied load distribution uncertainties than is the quasi-isotropic shell.

\section{Predicted and Measured Response Comparisons}

Selected results from nonlinear analyses of the orthotropic and quasi-isotropic shells are compared to the experimentally measured results in this section. The nonlinear analysis results are for shell models that included the effects of the measured initial shell-wall geometric and thickness imperfections, thickness-adjusted material property variations, shell-wall lamina ply-gaps, measured loading variations, elastic radial support conditions, and selected specimen parametric uncertainties. A value of the elastic radial support stiffness equal to $1.0 \mathrm{E} 5 \mathrm{lb} / \mathrm{in}$. is used in the models to simulate the boundary conditions provided by the pottedend supports of the shell specimens. Upper and lower 
response bounds were determined based upon the results of a traditional combinatorial analysis of the effects of selected specimen parametric uncertainties. Uncertainties in the measured thickness imperfection, lamina fiber volume fraction, and applied load distribution were considered in the analysis. Predicted and measured load-end-shortening response curves, selected load-strain responses, and observed and predicted post-collapse normal displacements are presented in this section.

Analytically predicted and experimentally measured load-end-shortening response curves for shells $\mathrm{C} 1, \mathrm{C} 2$ and $\mathrm{C} 3$ are presented in Fig. 26. Values of the axial load $P$ and the end-shortening $\Delta$ are normalized by the linear bifurcation buckling load of quasi-isotropic shell C3, $P_{b \text { if }}^{\text {quasi }}=42,590 \mathrm{lbs}$, , and the nominal shellwall thickness $t_{n o m}=0.04$ in., respectively. The solid and dashed lines in the figure represent experimentally measured and analytically predicted results, respectively. Each shell has two predicted response curves representing analytically predicted upper and lower bounds to the response based on specimen parametric uncertainties. The measured results indicate that shells $\mathrm{Cl}$, $\mathrm{C} 2$ and $\mathrm{C} 3$ exhibit the general instability points shown in the figure at normalized load values of $0.652,0.749$ and 0.803 , respectively, and are $7.8,13.7$ and $17.6 \%$ lower than the predicted loads for the geometrically perfect nominal shells shown in Fig. 9a, respectively. These results indicate that, for the most part, the measured load-end-shortening response curves for the shells fall on or within the analytically predicted response bounds. These results indicate that the measured response curves tend to correlate well with the analytically predicted lower bounds for each shell. Analytically predicted and experimentally measured strain response curves for selected points on shell C3 are presented in Figs. 27a and 27b. Values of the axial load $P$ and the axial strain $\varepsilon_{x}$ are normalized by the linear bifurcation buckling load of the quasi-isotropic shell $\mathrm{C} 3$, $P_{b i f}^{q u a s i}=42,590 \mathrm{lbs}$, and the corresponding axial strain at buckling $\varepsilon_{c r}=0.00257$, respectively. The strain results in both figures represent back-to-back surface strain measurements within the bending boundary layer near the ends of the shell. Strain gages 79 and 80 are outer and inner surface strain gages located at $x / L T_{T}=$ 0.071 and $\theta=0^{\circ}$, and strain gages 83 and 84 are outer and inner surface strain gages located at $x / L T=0.071$

and $\theta=180^{\circ}$. The bending boundary layer behavior is evident in the divergent character of the back-to-back predicted and measured strain results. These results indicate that the measured prebuckling strain responses, for the most part, fall on or within the predicted re- sponse bounds. However, there are some discrepancies in the predicted and measured post-collapse strain responses that are discussed subsequently. The results also indicate that the predicted post-collapse strain response can be very sensitive to specimen parametric uncertainties as shown in Fig. 27b. The measured and predicted prebuckling strain results for orthotropic shells C 1 and C2 agree well overall, but there are some discrepancies in the post-collapse predictions.

Predicted initial post-collapse normal displacement contours and the comesponding observed moiré fringe patterns for specimen $\mathrm{Cl}$ are shown in Fig. 28. The dashed contour lines in the predicted displacement contour plots represent inward displacements and the solid lines represent outward displacements. The density of the contour lines indicates the severity of the displacement gradients in the specimen. These results indicate that the specimen collapses into a general-instability diamond-shaped buckling pattern with 16 halfwaves around the circumference and one half-wave along the length, as predicted by the transient analysis. The analytical results predict that the mode-shape pattern is $15-20^{\circ}$ out-of-phase with the observed modeshape pattern. Predicted initial post-collapse normal displacement contours and the corresponding observed moiré fringe patterns for specimen $\mathrm{C} 2$ are shown in Fig. 29. These results indicate that the specimen collapses into a general instability diamond-shaped buckling pattern with 14 half-waves around the circumference and two half-waves along the length, as predicted by the transient analysis. In addition, the predicted modeshape is in-phase with the observed mode-shape. Similar results for shell $\mathrm{C} 3$ indicate that the shell collapses into a general-instability diamond-shaped pattern with 16 circumferential half-waves and two axial halfwaves. However, the analytical results predicted that the mode-shape pattern is approximately $15^{\circ}$ out-ofphase with the observed moiré fringe pattern. This discrepancy explains, in part, the large discrepancies in the post-collapse strain response indicated in Fig. 27.

Analytically predicted and experimentally measured load-end-shortening response curves for the 8 ply quasi-isotropic shell $\mathrm{C} 3$ and the 16-ply quasi-isotropic shell $\mathrm{C} 4$ are shown in Fig. 30. The axial load $P$ is normalized by the quantity $E A$, i.e., the effective axial stiffness of the shell, denoted by $E$, multiplied by the nominal shell cross-sectional area, denoted by $A$, and the end-shortening $\Delta$ is normalized by the nominal shell length $L=16.0 \mathrm{in}$. The solid and dashed lines in the figure represent experimentally measured and analytically predicted results, respectively. Each shell has two predicted response curves representing analytically predicted upper and lower bounds to the response based 
on specimen parametric uncertainties. The analytical results predict that shell $\mathrm{C} 4$ will buckle at an effective axial strain value $(\Delta L)$ of approximately 0.0052 0.0054 , which is approximately twice the value predicted for shell $\mathrm{C} 3$. The results for shell $\mathrm{C} 3$ indicate that for the most part, the measured load-end-shortening response curve for shell $\mathrm{C} 3$ fall on or within the analytically predicted response bound, however. The measured results for shell $\mathrm{C} 4$ fall outside of the predicted bounds for effective strain values greater than approximately 0.003 , and the shell has a limit load that is $10 \%$ lower than the predicted lower bound buckling load for the shell. The measured limit load for shell C4 corresponds to the complete failure of the shell and is characterized by significant material damage around the circumference of the shell. This shell had no postfailure residual strength. Post-test inspection of the shell indicated that the overall failure of the shell may have been initiated by a material failure near a circumferentially aligned ply-gap in the shell wall. To investigate that character of a possible material failure, a two-dimensional generalized plane-strain model of the ply-gap feature was developed to predict the local stress distribution around the ply-gap. The ply-gap model geometry and loading conditions are shown in Fig. 31a. The local ply-gap feature is subjected to a combined loading condition which is predicted by the global finite-element analysis of the entire shell. The loads applied to the ply-gap model are representative of the local loads near the ply-gap at buckling. The applied loads include an axial strain $\varepsilon_{x}$ equal to 0.005 , a bending rotation $r_{y}$ equal to 0.0002 radians, and a transverse shear load $Q_{x}$ equal to $20.0 \mathrm{lbf} / \mathrm{in}$. The predicted axial normal stress $\sigma_{x}$, transverse normal stress $\sigma_{z}$, and transverse shear stress $\sigma_{\mathrm{xz}}$ from the analysis are shown in Fig $31 \mathrm{~b}, \mathrm{c}$ and $\mathrm{d}$, respectively. The results indicate that the ply-gap causes significant stress concentrations to develop within the laminate. More specifically, the $0^{\circ}$ lamina ply near the ply-gap and the $0^{\circ}$ ply that exists towards the outer surface of the laminate have elevated values of all the stress components. The predicted results indicate that stress levels are equal to or exceed the typically reported stress allowalbles for this material system, and suggest that fiber compression failures and delamination failures could have occurred for these applied load values. In particular, several regions of the laminate have transverse shear stress values that are on the order of two to three times the allowable shear stress value for the material, and these results suggest that delamination failures near the ply-gap may have occurred for load values less than the predicted buckling load of the shell. These results suggest an explanation for some of the discrepancy between the predicted and measured results for shell $\mathrm{C} 4$.

\section{Design Considerations}

The nonlinear analysis procedure described herein offers a robust and accurate approach for predicting the nonlinear response and stability characteristics of compression-loaded thin-walled composite shell structures. This nonlinear analysis procedure can be used to form the basis of a modern high-fidelity design and analysis approach for composite shell structures that accounts for the effects of imperfections associated with the fabrication process used to fabricate the structure. A comparison of results from the traditional shell design approach and from the present nonlinear high-fidelity shell analysis approach is shown in Fig. 32 for shell C3. Values of the axial load $P$ and the end-shortening $\Delta$ in the figure are normalized by the predicted linear bifurcation buckling load for quasi-isotropic shell C3, $P_{\text {bif }}^{q u n s i}=42,590 \mathrm{lbs}$, and the nominal shell-wall thickness $t_{\text {nom }}=0.04$ in., respectively. The traditional approach to shell design is to predict the shell buckling load using a linear bifurcation buckling analysis with the nominal structural dimensions and material properties of an idealized geometrically prefect shell. This predicted bucking load for the shell is then reduced by an empirical "knockdown" factor based on a design criterion such as the lower-bound design recommendations reported in NASA SP-8007 (Ref. 1). Quasiisotropic shell $\mathrm{C} 3$ has a nominal radius-to-shell-wallthickness ratio equal to 200, and the design knockdown factor from NASA SP-8007 for an equivalent isotropic shell is approximately equal to 0.47 as indicated in Fig. 32. This traditional design approach results in an overly conservative design for this shell. The normalized measured buckling load and the normalized predicted buckling load from the lower response bound predicted by the nonlinear analysis procedure are 0.803 and 0.832 , respectively, and these results are approximately 33 and $36 \%$ greater than the results based on the traditional design approach. The modern high-fidelity analysis approach offers a relatively affordable alternative to relying on historical test data for shells that do not represent the configuration, material system, or fabrication process for a particular composite shell design of interest. This suggested design analysis procedure should be used with a selected number of carefully conducted experiments that could be used to verify the design and analysis results. This approach could make it possible to avoid testing the large number of replicates of a design needed to develop empirical design factors. This nonlinear analysis procedure could be used as a parametric tool in the early stages of a design development program to determine the sensitivity of the response 
program to determine the sensitivity of the response characteristics of a specific design to a number of different types of imperfections or differences in the idealized as-designed shell structure and the actual asmanufactured shell structure. Since this nonlinear analysis procedure can predict the local stresses and strains in a shell for any point in the load-response history of the shell, failure of the shell due to local stress or strain gradients could also be predicted.

\section{Concluding Remarks}

The results of an experimental and analytical study of the effects of imperfections on the nonlinear response and buckling loads of unstiffened thin-walled compression-loaded graphite-epoxy cylindrical shells with four shell-wall laminates are presented. The shellwall laminates considered in this study include two quasi-isotropic laminate and two different orthotropic laminates. Shell-radius-to-thickness ratios equal to 100 and 200 were also considered. The results for the nonlinear prebuckling, buckling, transient collapse, and post-collapse response of geometrically perfect shells and shells with measured imperfections are presented. The results identify the effects of traditional initial geometric shellwall mid-surface imperfections and the effects of other nontraditional imperfections on the nonlinear response and buckling loads of the shells. These nontraditional imperfections include shell-wall thickness variations, material property variations, shell-end geometric imperfections, local shell-wall ply-gaps associated with the fabrication process, variations in loads applied to the end of the shell, and elastic boundary support conditions. In addition, upper and lower bounds to the nonlinear response of the shells are presented which were determined from a combinatorial analysis of the effects of uncertainties or variations in several shell parameters. A high-fidelity nonlinear shell analysis procedure has been used to predict the nonlinear response of the shells, and the analysis procedure accurately accounts for the effects of these traditional and nontraditional imperfections and elastic boundary conditions on the nonlinear response and buckling loads of the shells. The analysis results generally correlate well with the experimental results indicating that it is possible to predict accurately the complex nonlinear response and buckling loads for compression-loaded composite shell structures when the traditional and nontraditional imperfections considered in the present study are included in the analysis.

The analytical results indicate that the effects of the traditional and nontraditional imperfections considered in this study can be important for predicting the buckling loads of composite shells since they can significantly affect the nonlinear response and buckling loads of the shells. The results indicate that the measured imperfections can couple with the in-plane compressive stress resultants in a nonlinear manner to affect the shell response. The results show that shell-wall laminate orthotropy can also have a significant effect on the nonlinear response and buckling loads of composite shell structures. However, the results indicate that the nonlinear response and buckling loads of the orthotropic shells studied are less sensitive to the measured imperfections considered in this study than are the nonlinear response and buckling loads of the quasi-isotropic shell studied. In addition, the results indicate that elastic boundary support conditions can have a significant effect on the displacement and strain responses of the shells; shell length and shell-wall orthotropy can have a significant effect on the attenuation of the shellend bending boundary layer; and shell-wall ply-gaps associated with the fabrication process can have a significant effect on the shell buckling loads. Moreover, the results indicate that shell-wall ply-gaps can cause significant stress gradients to develop in the shell that, in turn, could cause premature failure of the shell.

The results indicate that, for the most part, the measured response of the shells falls on or within the predicted upper and lower bounds to the response that are associated with the uncertainties or variations in the shell parameters considered in the study. These results indicate that the nonlinear analysis procedure used in this study can be used to determine accurate, high-fidelity design knockdown factors that can be used for predicting composite shell buckling and collapse loads in the design process. The traditional and nontraditional imperfections considered in this study could be used to formulate the basis for a generalized imperfection signature of a composite shell that includes the effects variations or uncertainties in the shell-geometry, fabrication-process, load-distribution and boundary stiffness parameters. The high-fidelity nonlinear analysis procedure used in this study can be used to form the basis for a shell analysis and design approach that includes this generalized imperfection signature and addresses some of the critical shell-buckling design criteria and design considerations for composite shell structures without resorting to the traditional empirical shell design approach that can lead to overly conservative designs. Since the nonlinear analysis procedure can be used to predict local shell-wall stresses and strains at any point in the shell load-response history, the analysis procedure can also be used to form a robust failure analysis for composite shell structures with nonlinear response characteristics. 


\section{References}

1. Anon., Buckling of Thin-Walled Circular Cylinders. NASA Space Vehicle Design Criteria, NASA SP-8007, September 1965.

2. Koiter, W. T., "On the Stability of Elastic Equilibrium," (in Dutch), H. J. Paris, Amsterdam, Holland, 1945; translation available as AFFDL-TR-70-25, February 1970, Wright-Patterson Air Force Base.

3. von Kármán, T. and Tsien, H-S., "The Buckling of Thin Cylindrical Shells Under Axial Compression," Journal of the Aeronautical Science, Vol. 8, No. 8, June 1941, pp. 303-312.

4. Budiansky, B. and Hutchinson, J., "Dynamic Buckling of Imperfection Sensitive Structures," Proceedings of the 11 th IUTAM Congress, H. Gortler, Ed., Springer-Verlag, Berlin, 1964, pp. 636-651.

5. Árbocz, J. and Babcock, C. D., "The Effect of General Imperfections on the Buckling of Cylindrical Shells," Journal of Applied Mechanics, Vol. 36, Series E, No. 1, 1969, pp. 28-38.

6. Sechler, E. E., "The Historical Development of Shell Research and Design," in Thin-Shell Structures, Theory, Experiments and Design, Fung, Y. C. and Sechler, E. E., Eds., Prentice-Hall, Englewood Cliffs, NJ, 1974, pp. 3-25.

7. Árbocz, J., "The Effects of Imperfect Boundary Conditions on the Collapse Behavior of Anisotropic Shells," Proceedings of the Joint Applied Mechanics and Materials ASME Summer Conference, AMDMD'95, Los Angeles, CA, June 28-30, 1995.

8. Árbocz, J., Starnes, J. H., Jr., and Nemeth, M. P., "A Hierarchical Approach to Buckling Load Calculations," Proceedings of the 40th AIAA/ASME/ASCE/ AHS/ASC Structures, Structural Dynamics, and Materials Conference, St. Louis, MO, 1999. AIAA Paper No. 99-1232, April 1999.

9. Koiter, W. T., Elishakoff, I., Li, Y. W., and Starnes, J. H., Jr., "Buckling of an Axially Compressed Imperfect Cylindrical Shell of Variable Thickness," Proceedings of the 35th AIAA/ASME/ASCE/AHS/ ASC Structures, Structural Dynamics and Materials Conference, Hilton Head, SC, 1994. AIAA Paper No. 94-1339, April 1994.
10. Starnes, J. H., Jr., Hilburger, M. W., and Nemeth, M. P., "The Effects of Initial Imperfections on the Buckling of Composite Shells," Composite Structures: Theory and Practice, ASTM STP 1383, P. Grant and C. Q. Rousseau, Eds., American Society for Testing and Materials, 2000, pp. 529-550.

11. Hilburger, M. H., and Starnes, J. H., Jr., "Effects of Imperfections on the Buckling Response of Compression-loaded Composite Shells," Proceedings of the 41st AIAA/ASME/ASCE/AHS/ASC Structures, Structural Dynamics, and Materials Conference, Atlanta, GA, 2000. AIAA Paper No. 2000-1387, April 2000.

12. Holst, J. M., Rotter, J. M., and Calladine, C. R., "Imperfections in Cylindrical Shells Resulting from Fabrication Misfits," Journal of Engineering Mechanics, Vol. 125, No. 4, 1999, pp. 410-418.

13. Rankin, C. C., Brogan, F. A., Loden, W. A., and Cabiness, H. D., "STAGS Users Manual, Version 3.0," Lockheed Martin Missiles \& Space Co., Inc., Advanced Technology Center, Palo Alto, CA, Report LMSC P032594, 1999.

14. Riks, E., "Progress in Collapse Analysis,"Journal of Pressure Vessel Technology, Vol. 109, 1987, pp. 27-41.

15. Park, K. C., "An Improved Stiffly Stable Method for Direct Integration of Nonlinear Structural Dynamics," Journal of Applied Mechanics, Vol. 42, June 1975, pp. 464-470.

16. Riks, E., Rankin, C. C., and Brogan, F. A., "On the Solution of Mode Jumping Phenomena in Thinwalled Shell Structures," Computer Methods in Applied Mechanics and Engineering, Vol. 136 (1-2), 1996, pp. 59-92.

17. Weiland, T. M., Morton, J., and Starnes, J.H., "Scaling Effects in Buckling, Postbuckling and Crippling of Graphite-Epoxy Z-Section Stiffeners," Virginia Polytechnic Institute and State University, Center for Composite Materials and Structures, Report CCMS-9225, September 1992.

18. Fuchs, J. P., Hyer, M. W., and Starnes, J. H., "Numerical and Experimental Investigation of the Bending Response of Thin-Walled Composite Cylinders," Virginia Polytechnic Institute and State University, Center for Composite Materials and Structures, Report CCMS-93-19/VPI-E-93-11, September 1993. 


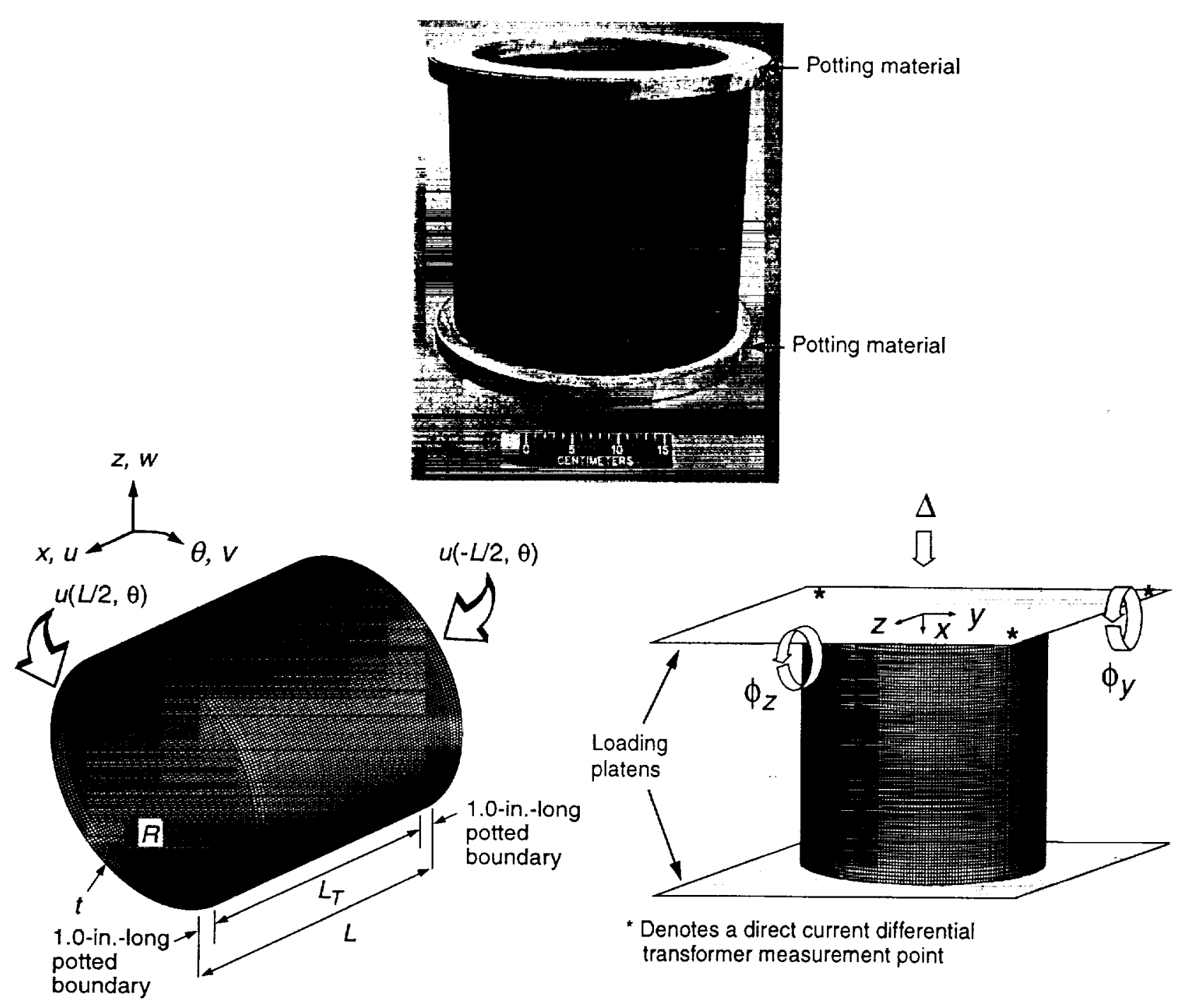

Fig. 1 Typical specimen, finite-element model geometry and loading conditions.

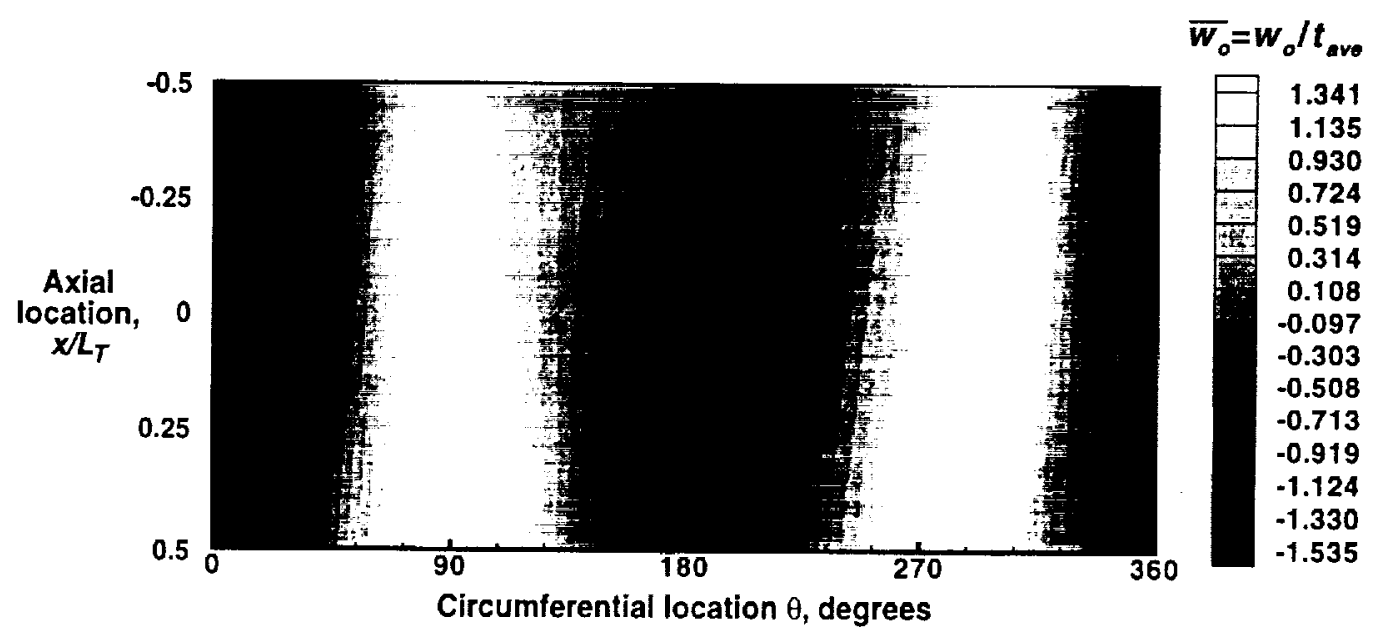

Fig. 2 Typical measured inner-surface imperfection shape for shell specimen $\mathbf{C 3}$. 


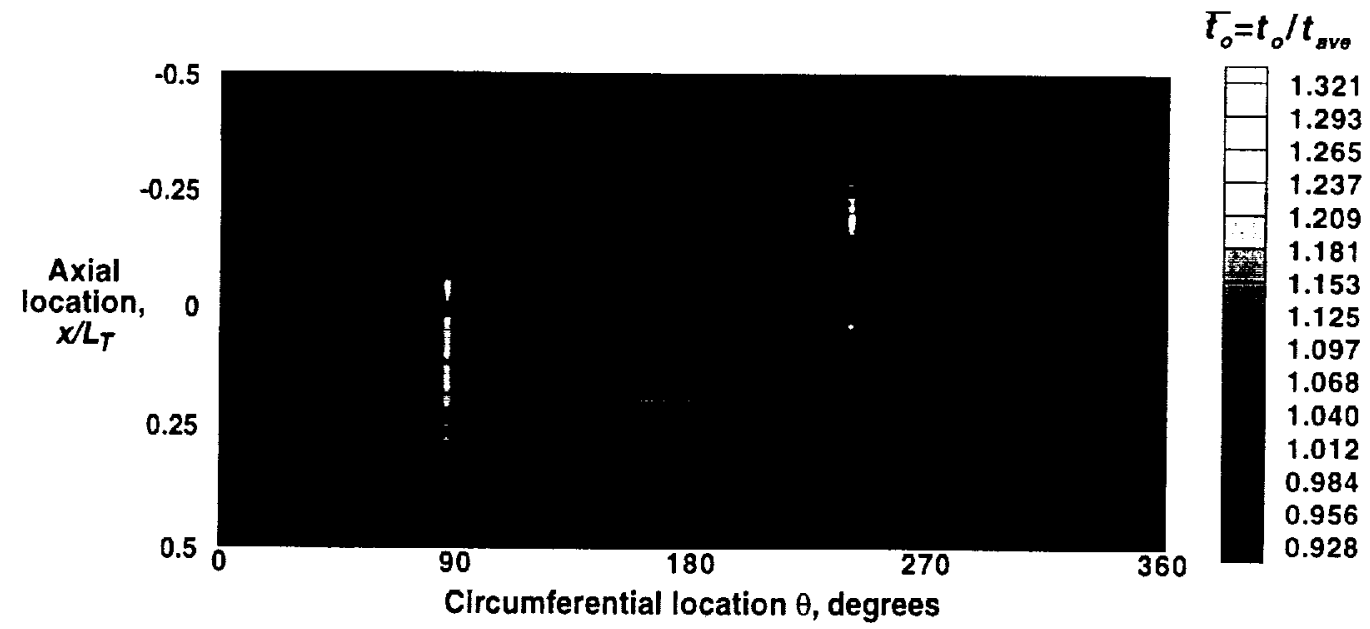

Fig. 3 Typical measured wall thickness variation for a shell specimen C3.

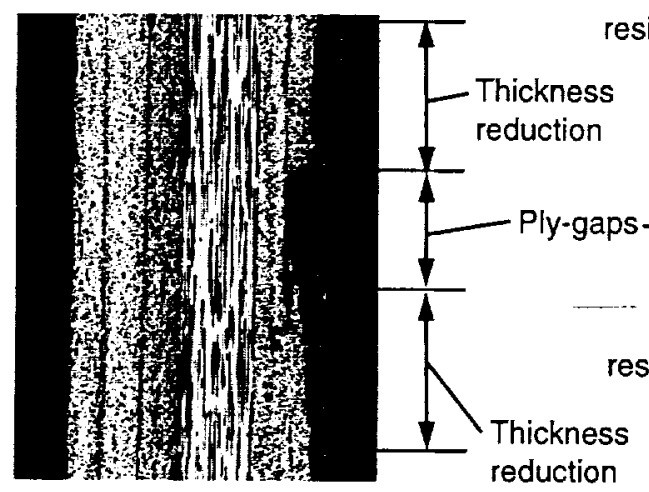

a) Ply-gap in a $45^{\circ}$ outersurface lamina ply

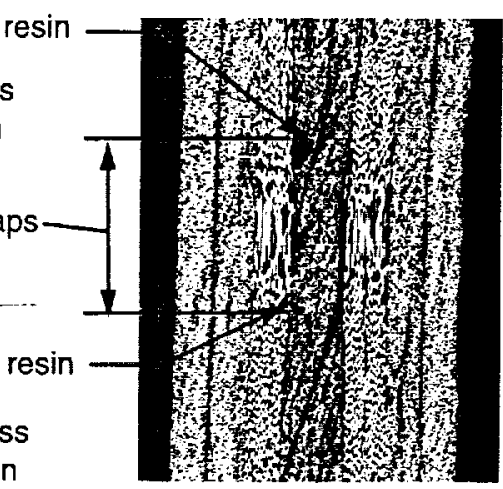

b) Ply-gap in a $90^{\circ}$ innersurface lamina ply

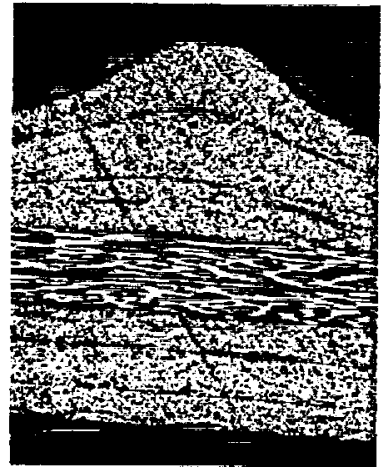

c) Outer-surface ridge

Fig. 4 Magnified cross-sectional views of lamina ply-gaps and a magnified cross-sectional view of an outer-surface ridge.
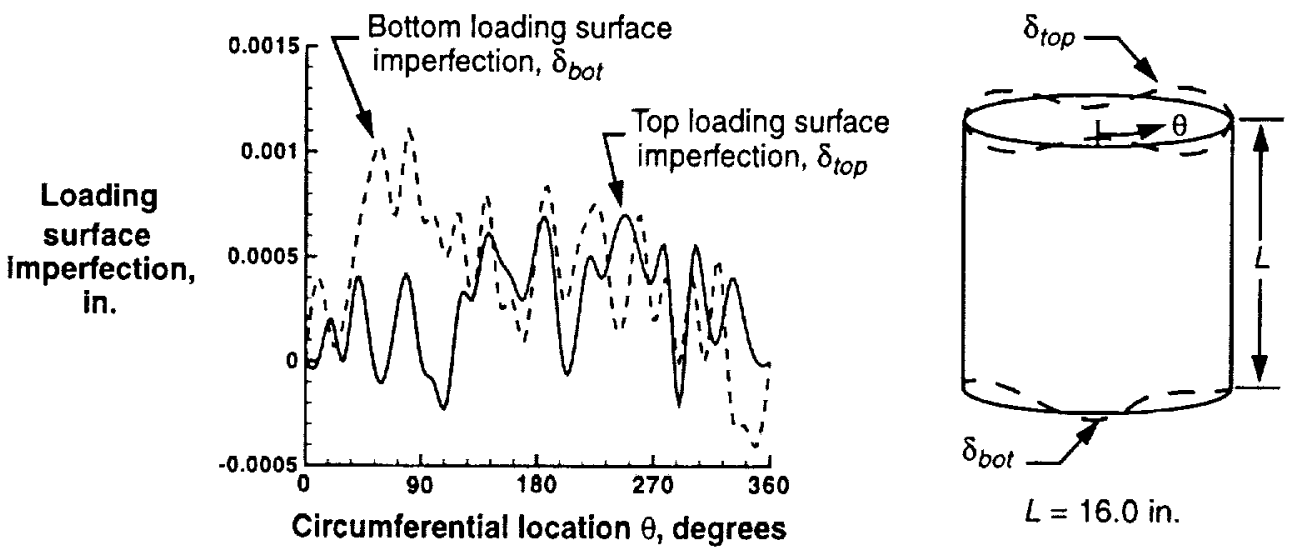

Fig. 5 Typical measured shell-end or loading-surface imperfections for a shell specimen C3. 


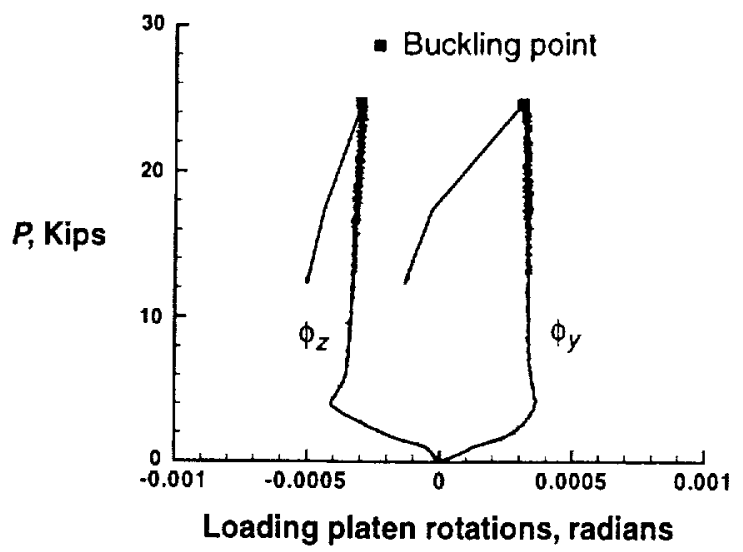

Fig. 6 Typical experimentally measured loading platen rotations.

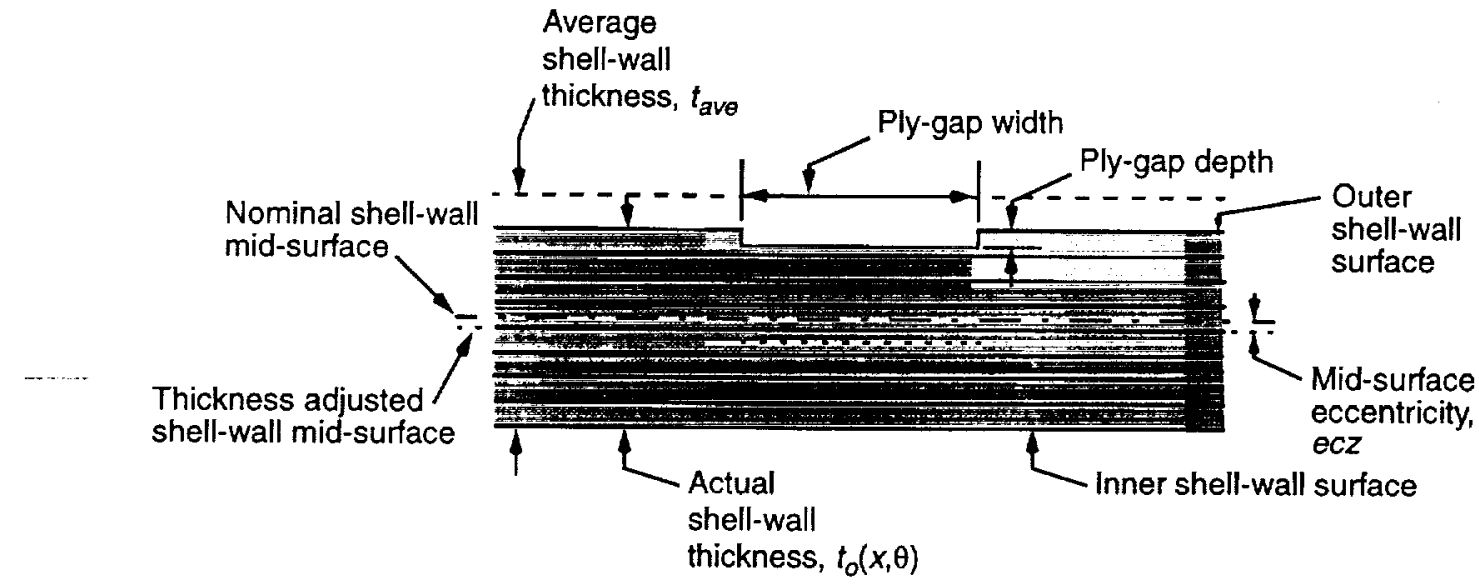

Fig. 7 Idealized ply-gap model detail.
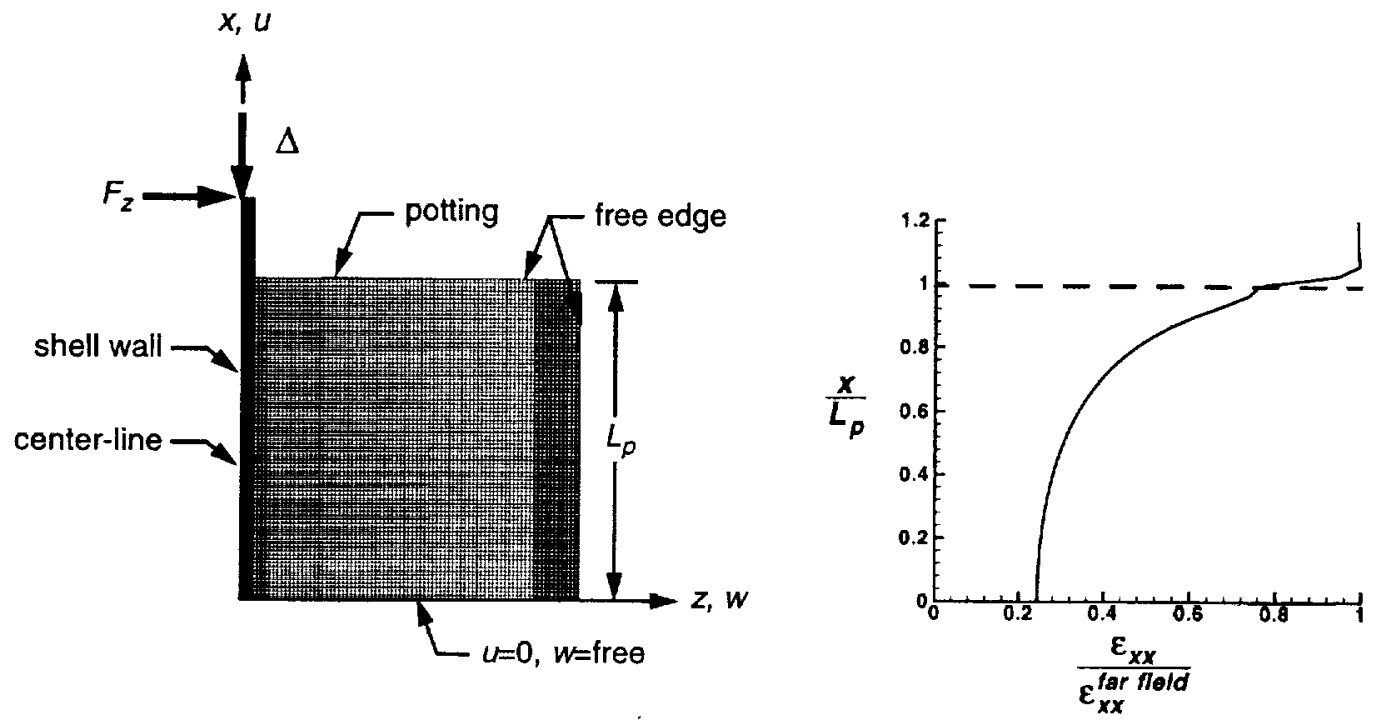

Fig. 8 Typical finite-element model and geometry and boundary conditions of the potting-shell detail and typical predicted axial strain distribution at the mid-surface of the shell wall. 


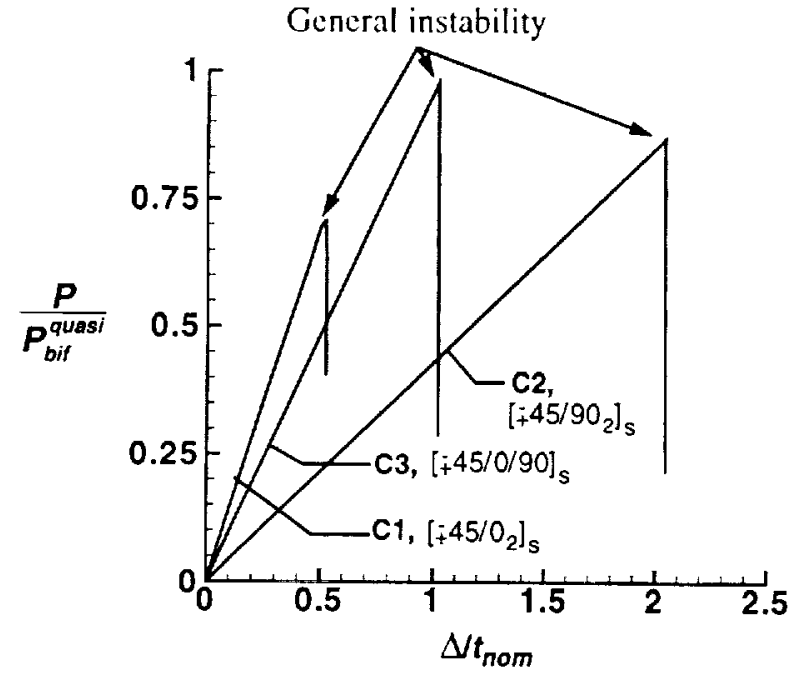

a) Normalized load-end-shortening response curves

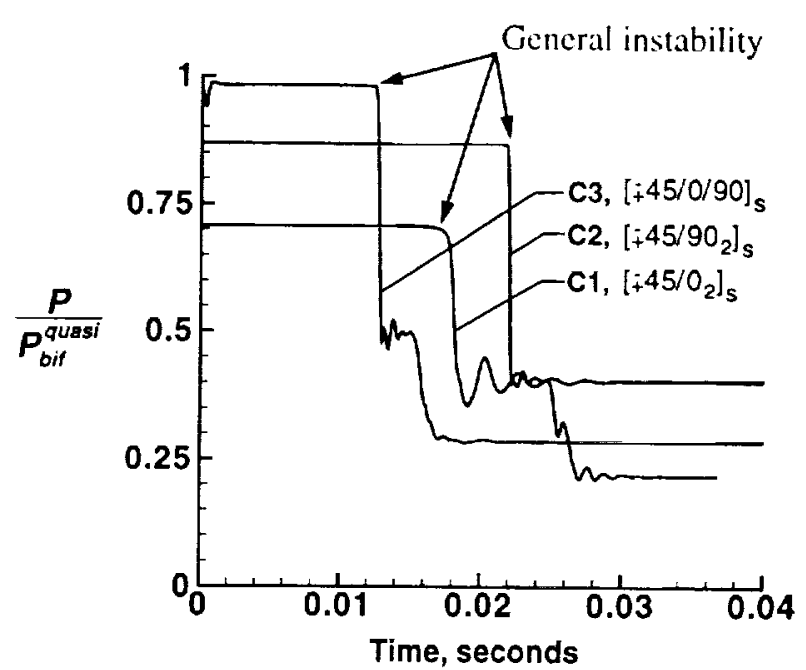

b) Normalized load-time history during collapse

Fig. 9 Numerically predicted nonlinear response of geometrically perfect compression-loaded cylindrical shells.

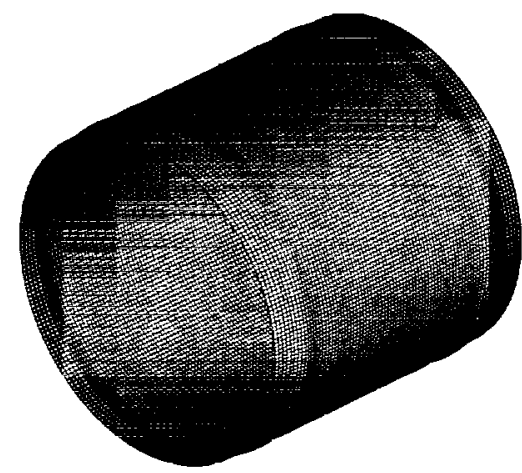

a) Prebuckling deformations Time $=0.0$ seconds $P / P_{\text {bif }}^{\text {quasi }}=0.979$

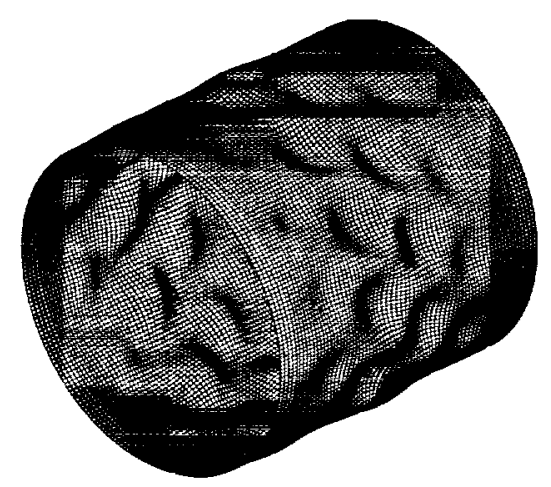

d) Transient collapse deformations Time $=0.0134$ seconds $P / P_{\text {hif }}^{\text {quasi }}=0.465$

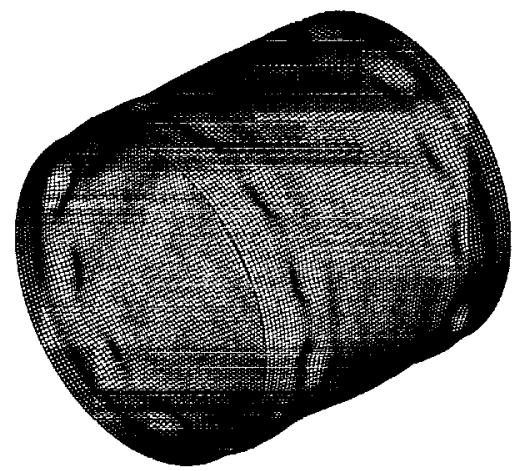

b) Initial collapse deformations Time $=0.0125$ seconds $P / P_{b i f}^{\text {quasi }}=0.983$

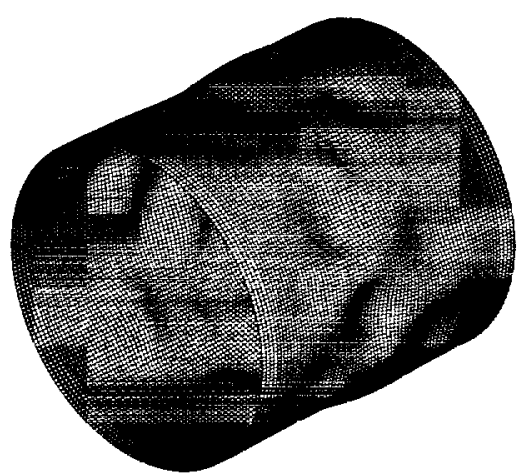

e) Transient collapse deformations Time $=0.0152$ seconds $P / P_{\text {lif }}^{\text {quasi }}=0.426$

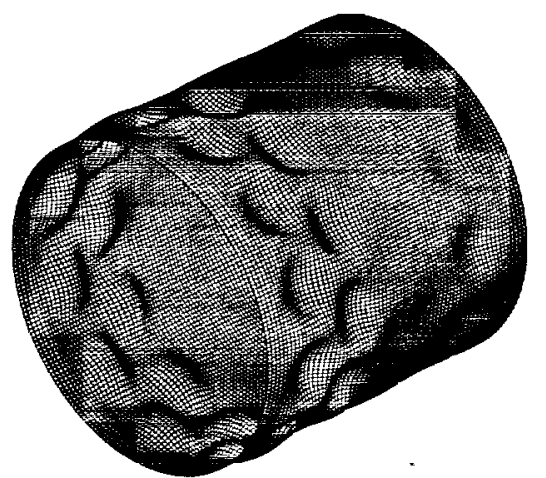

c) Transient collapse deformations Time $=0.0130$ seconds $P / P_{\text {bif }}^{\text {quasi }}=0.479$

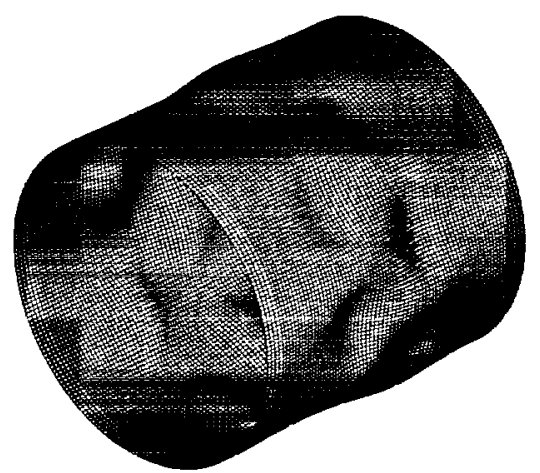

f) Post-collapse Time $=0.04$ seconds $P / P_{\text {by }}^{\text {quasi }}=0.286$

Fig. 10 Numerically predicted collapse response for a geometrically perfect compression-loaded quasi-isotropic shell C3. 


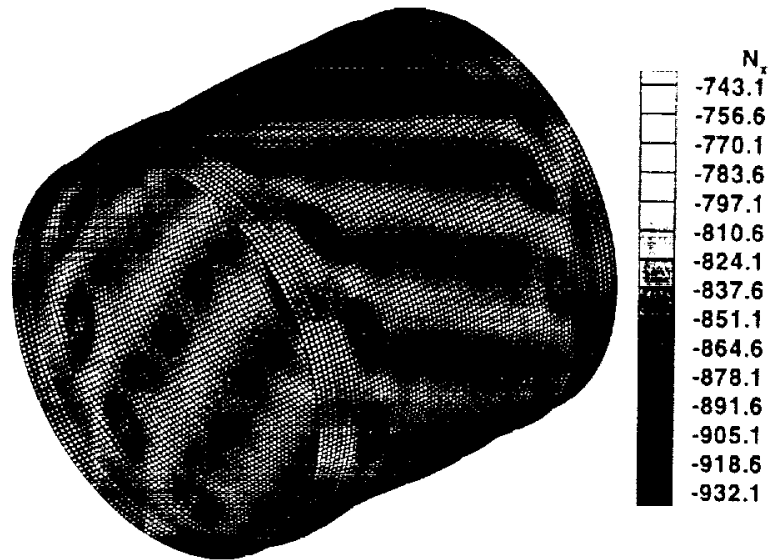

a) Axial stress resultant contours, $\mathrm{N}_{\mathrm{x}}$, Ibf/in

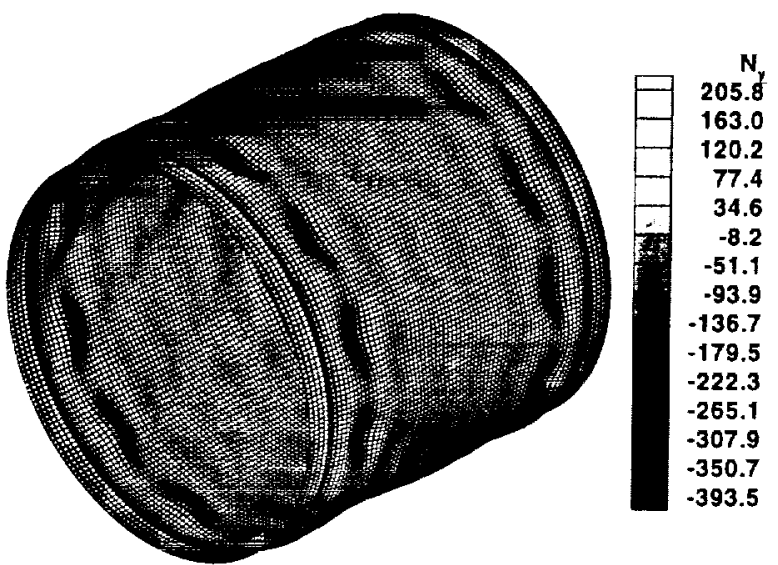

b) Circumferential stress resultant contours, $\mathrm{N}_{\mathrm{y}} \mathrm{lbf} / \mathrm{in}$

Fig. 11 Numerically predicted axial and circumferential stress resultants, $N_{x}$ and $N_{y}$, respectively, just before buckling for a geometrically perfect compression-loaded quasi-isotropic shell $\mathrm{C3}$.

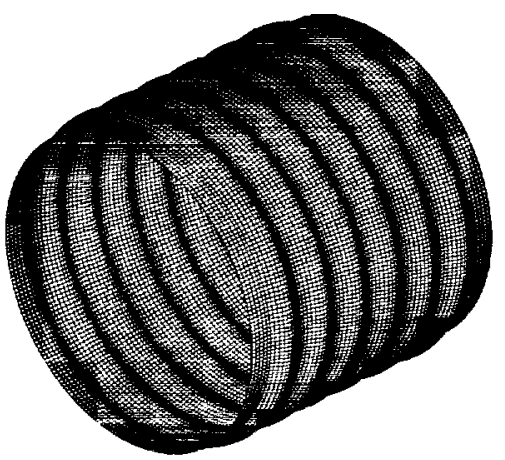

a) Prebuckling deformations

Time $=0.0$ seconds $P / P_{\text {bif }}^{\text {quasi }}=0.707$

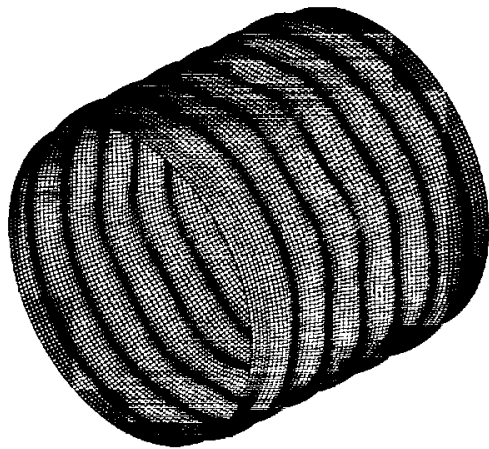

b) Initial collapse deformations Time $=0.0167$ seconds $P / P_{\text {bif }}^{\text {quasi }}=0.704$

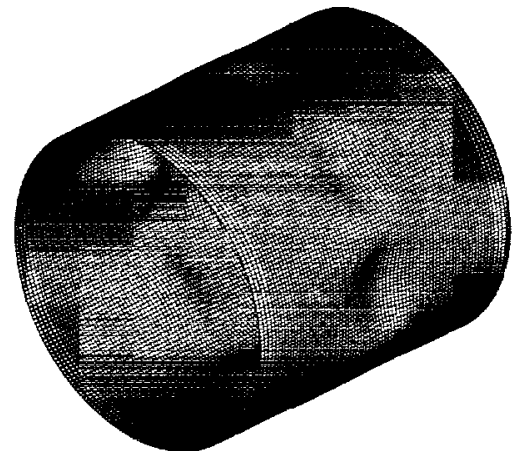

c) Post-collapse deformations Time $=0.04$ seconds $P / P_{\text {bif }}^{\text {qursi }}=0.403$

Fig. 12 Numerically predicted collapse response for a geometrically perfect compression-loaded orthotropic shell C1.

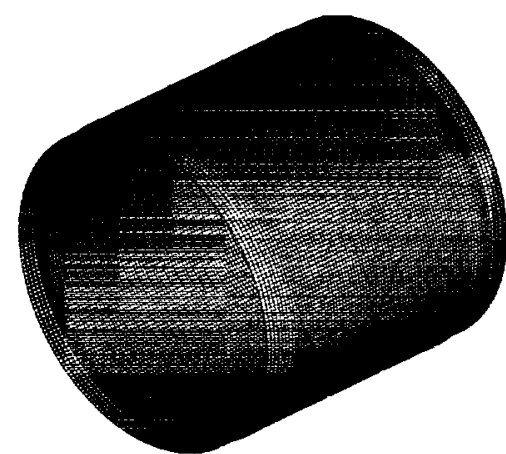

a) Prebuckling deformations Time $=0.0$ seconds $P / P_{\text {bif }}^{\text {quasi }}=0.868$

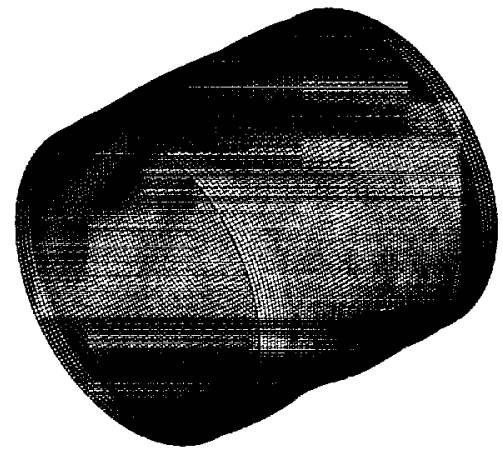

b) Initial collapse deformations Time $=0.0217$ seconds $P / P_{\text {bif }}^{\text {qusi }}=0.866$

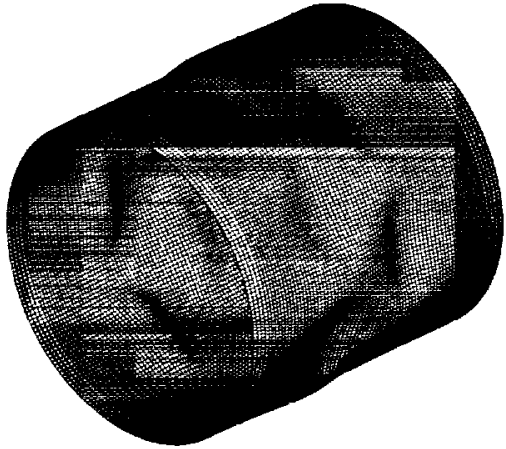

c) Post-collapse

Time $=0.037$ seconds $P / P_{\text {bif }}^{\text {quasi }}=0.218$

Fig. 13 Numerically predicted collapse response for a geometrically perfect compression-loaded orthotropic shell C2. 


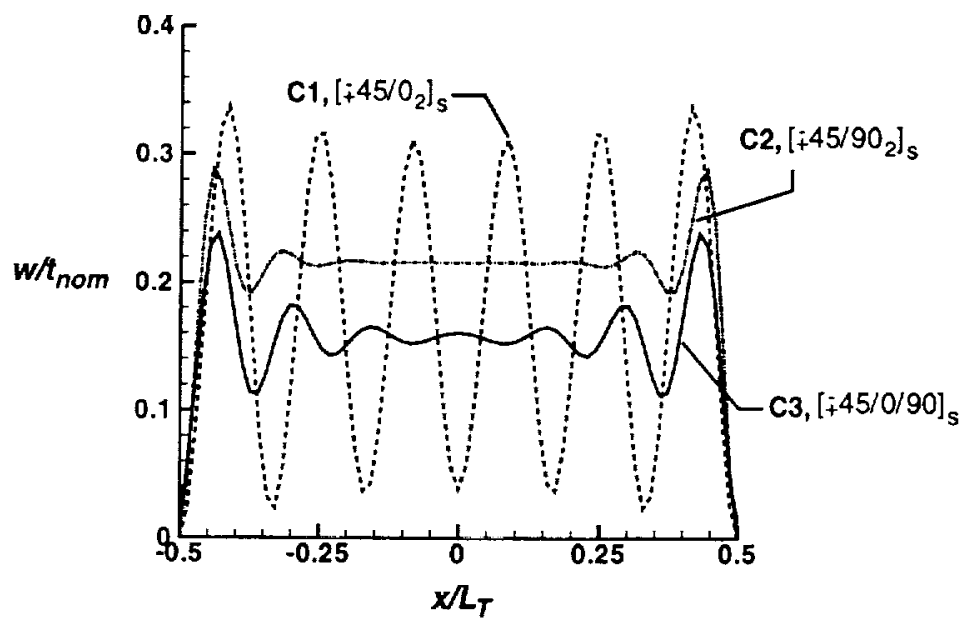

Fig. 14 Effects of orthotropy on displacements just before buckling of geometrically perfect compressionloaded composite cylindrical shells.

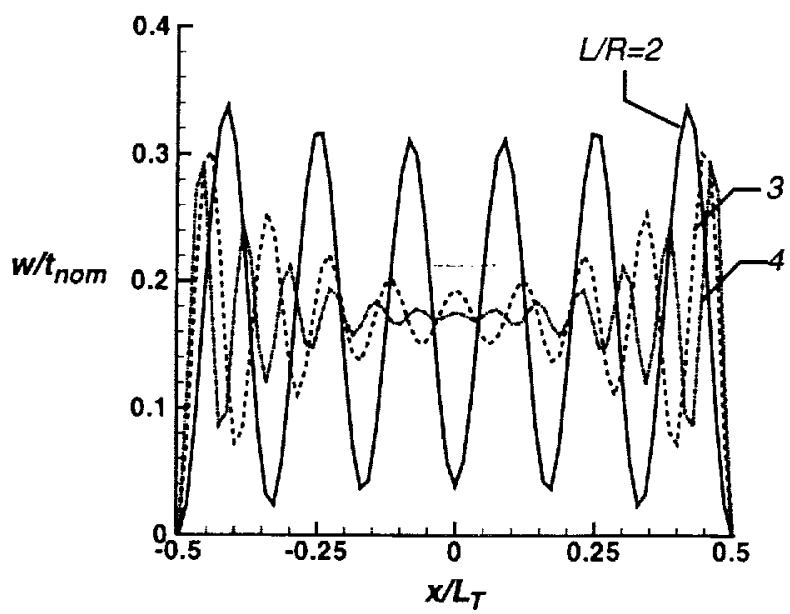

Fig. 15 Effects of shell length on bending boundary layer attenuation for a geometrically perfect compressionloaded orthotropic shell C1. 


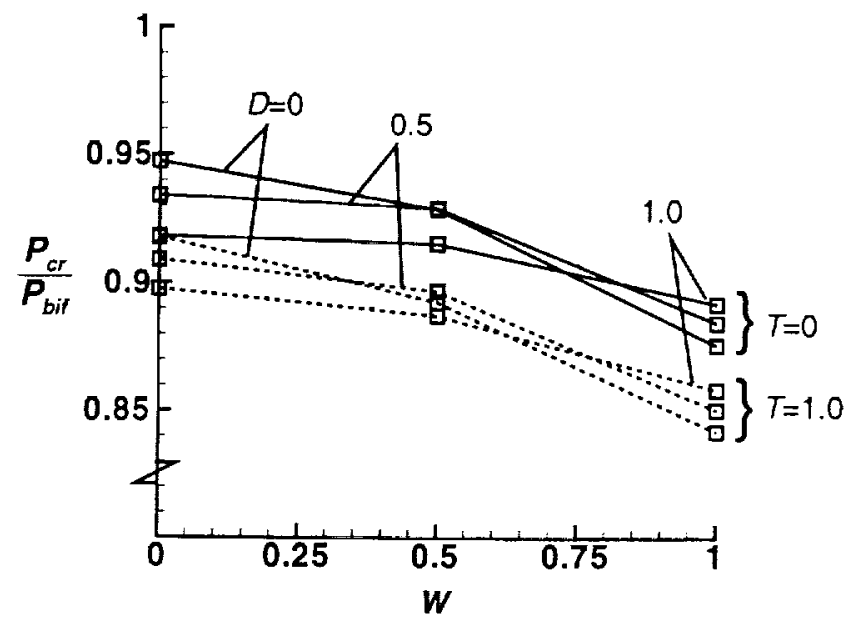

Fig. 16 Effects of measured imperfections on the normalized buckling load of a compression-loaded quasi-isotropic cylindrical shell C3. Imperfection amplitude scale factors for the measured geometric shell-wall midsurface imperfection, shell-wall thickness imperfection, and nonuniform Ioading are denoted by $W, T$, and $D$, respectively.

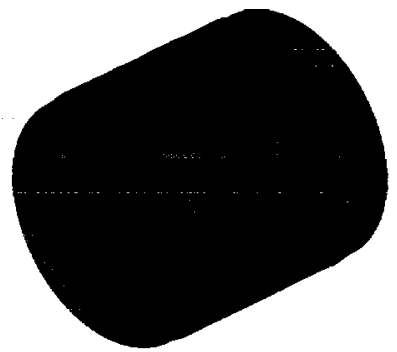

a) Prebuckling deformations Time $=0.0$ seconds $P / P_{c r}=0.858$

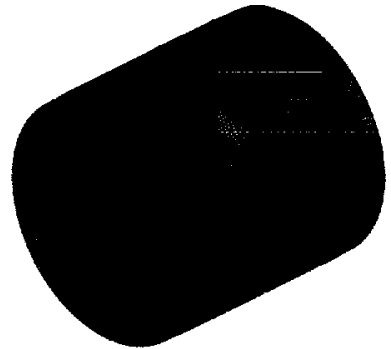

b) Initial collapse deformations Time $=0.00143$ seconds $P / P_{c r}=0.855$

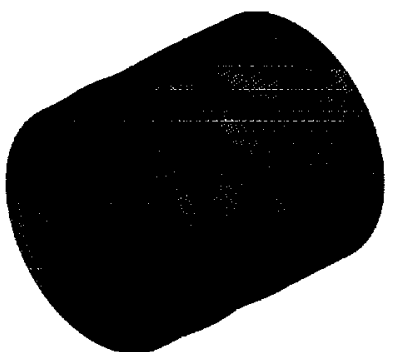

c) Transient collapse deformations

Time $=0.00238$ seconds $P / P_{c r}=0.598$

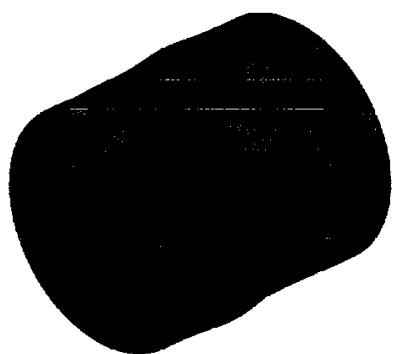

d) Post-collapse deformations Time $=0.02$ seconds $P / P_{c r}=0.311$

Fig. 17 Numerically predicted collapse response for an imperfect compression-loaded quasi-isotropic shell C3.

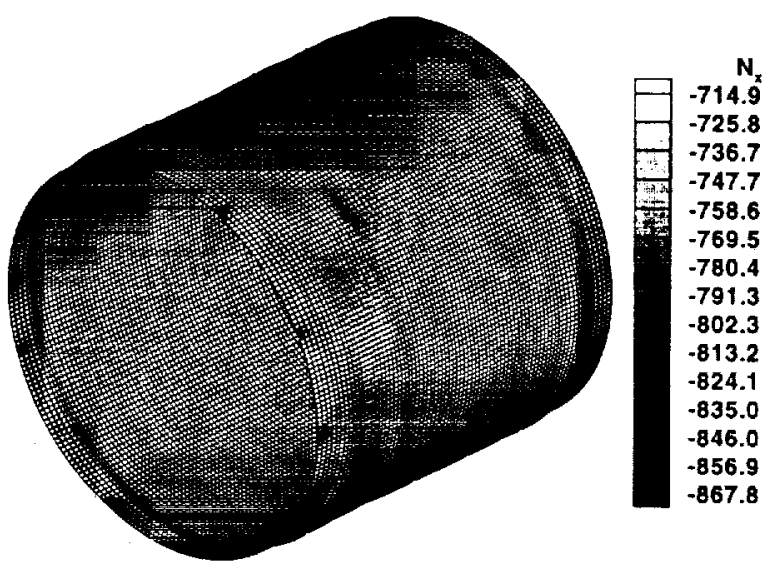

a) Axial stress resultant contours, $\mathrm{N}_{\mathrm{x}}$, lbf/in.

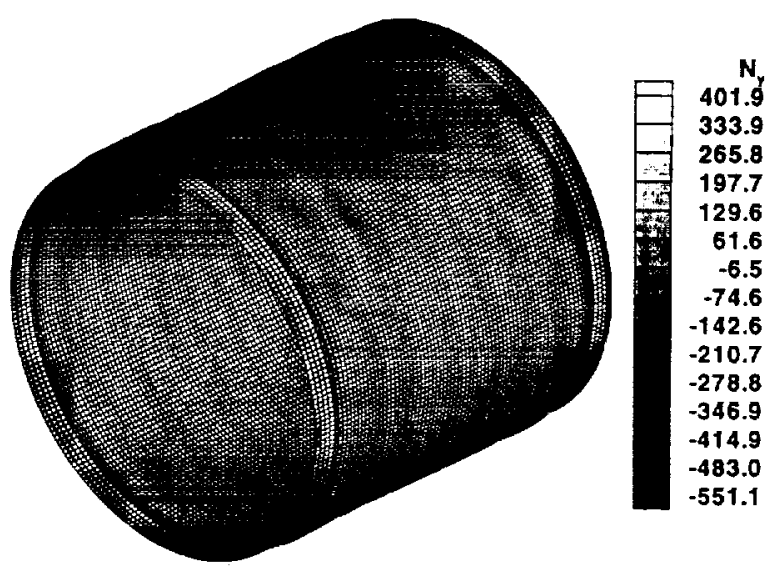

b) Circumferential stress resultant contours, $N_{y}$, lbf/in.

Fig. 18 Numerically predicted axial and circumferential stress resultants, $N_{x}$ and $N_{y}$, respectively, just before buckling for an imperfect compression-loaded quasi-isotropic shell C3. 


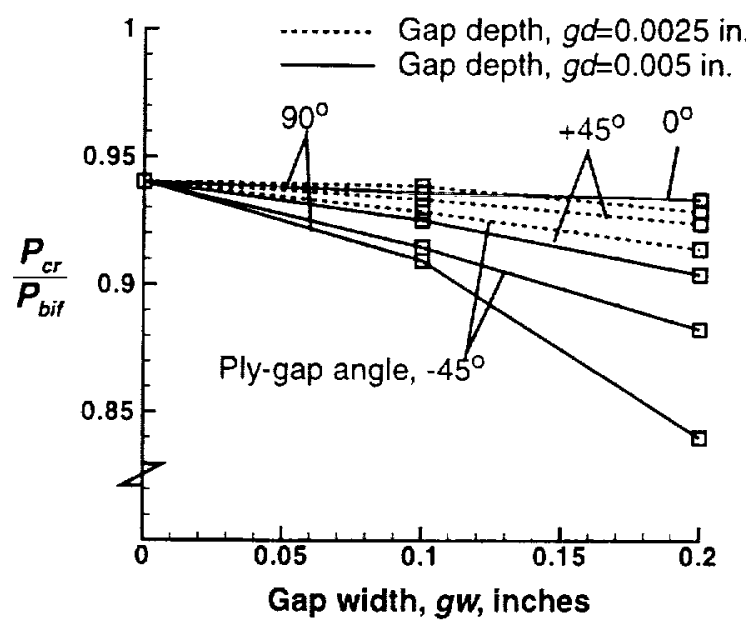

Fig. 19 Effects of ply-gap angle, gap width $g w$, and gap depth $g d$, on the normalized buckling load of a geometrically perfect compression-loaded quasi-isotropic cylindrical shell $\mathrm{C} 3$.

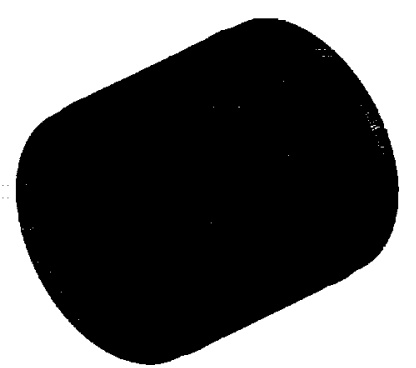

a) Prebuckling deformations Time $=0.0$ seconds $P / P_{c r}=0.887$

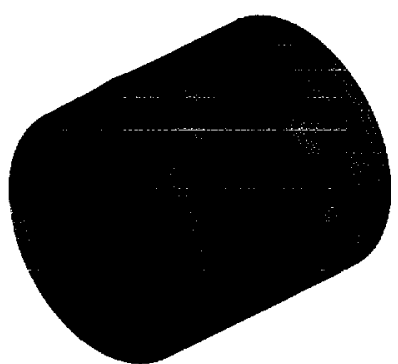

b) Initial collapse deformations Time $=0.0012$ seconds $P / P_{c r}=0.885$

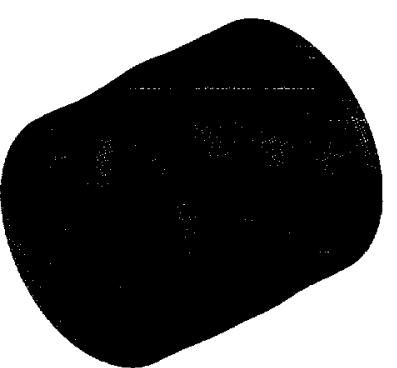

c) Transient collapse deformations Time $=0.0029$ seconds $P / P_{c r}=0.588$

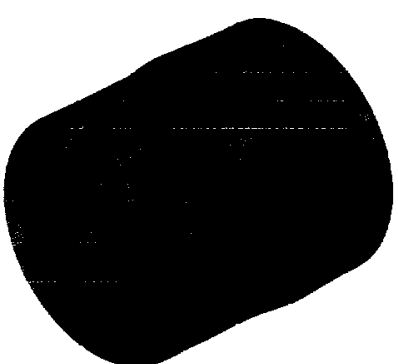

d) Post-collapse deformations Time $=0.02$ seconds $P / P_{c r}=0.310$

Fig. 20 Effects of a $45^{\circ}$ lamina ply-gap on the transient collapse response of a compression-loaded quasi-isotropic cylindrical shell C3.

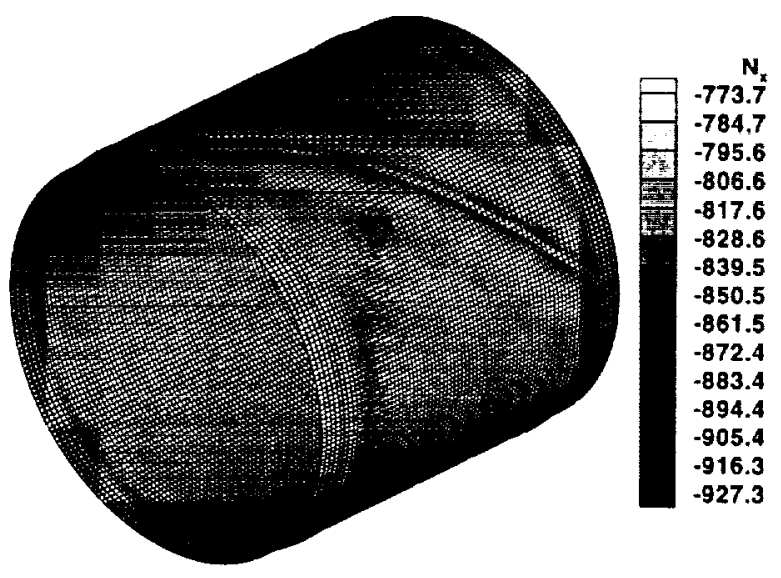

a) Axial stress resultant contours, $N_{x}, l b f / i n$.

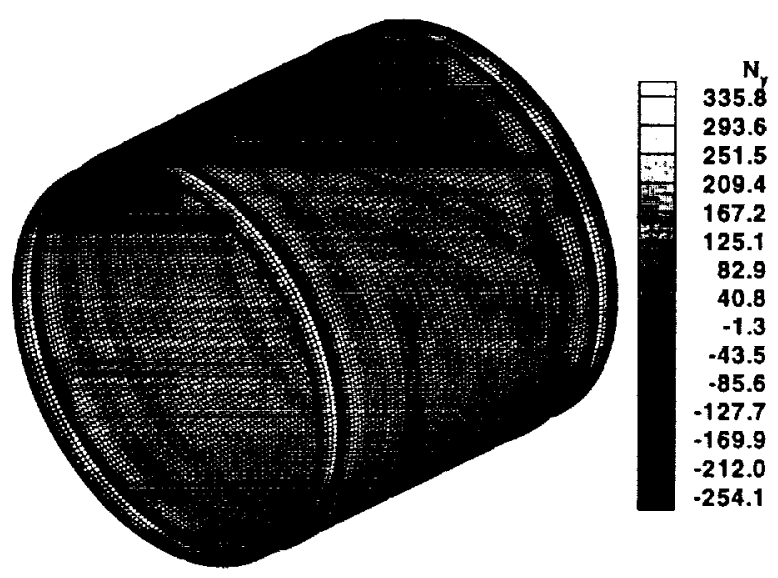

b) Circumferential stress resultant contours, $\mathrm{N}_{\mathrm{y}}, \mathrm{lbf} / \mathrm{in}$.

Fig. 21 Effects of a $45^{\circ}$ lamina ply-gap on the axial and circumferential stress resultants incipient to buckling for a compression-Ioaded quasi-isotropic cylindrical shell $\mathrm{C} 3$. 


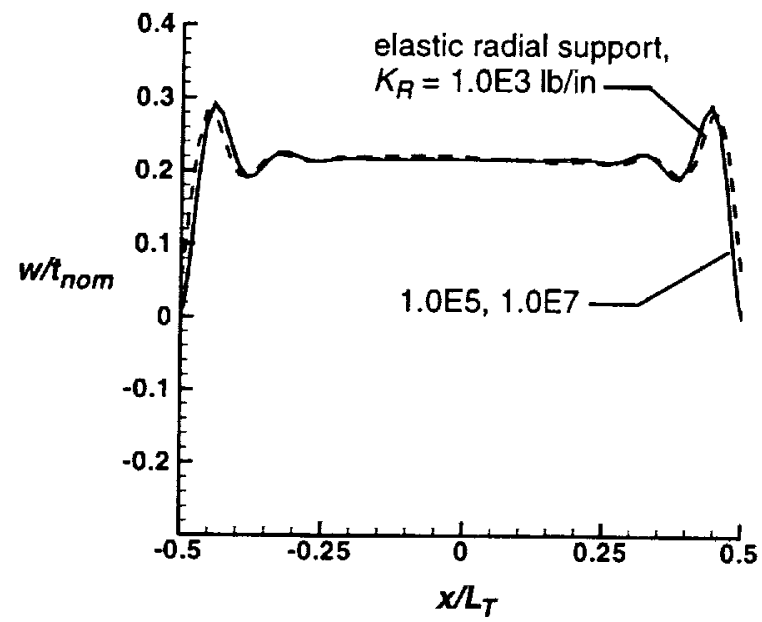

a) Geometrically perfect

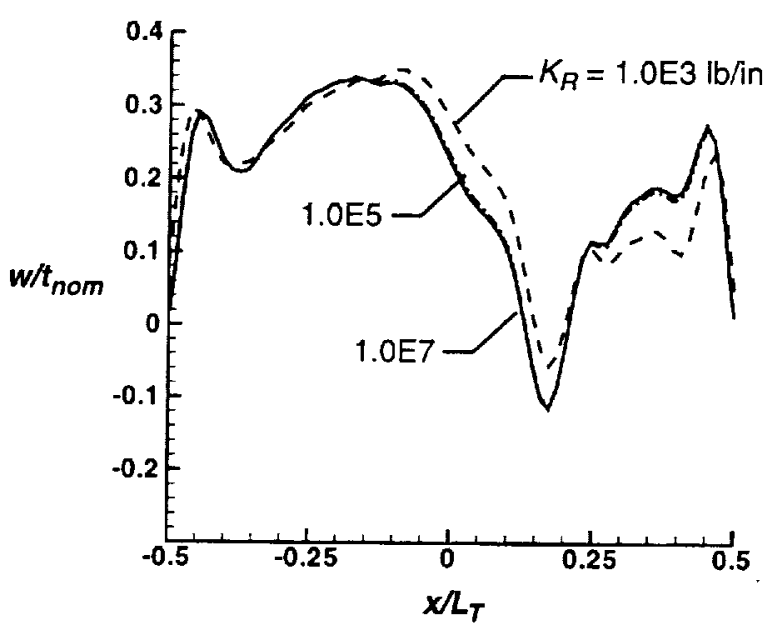

b) With measured imperfections

Fig. 22 Effects of elastic radial support conditions on the buckling displacement profile of compression-loaded orthotropic shell C2.

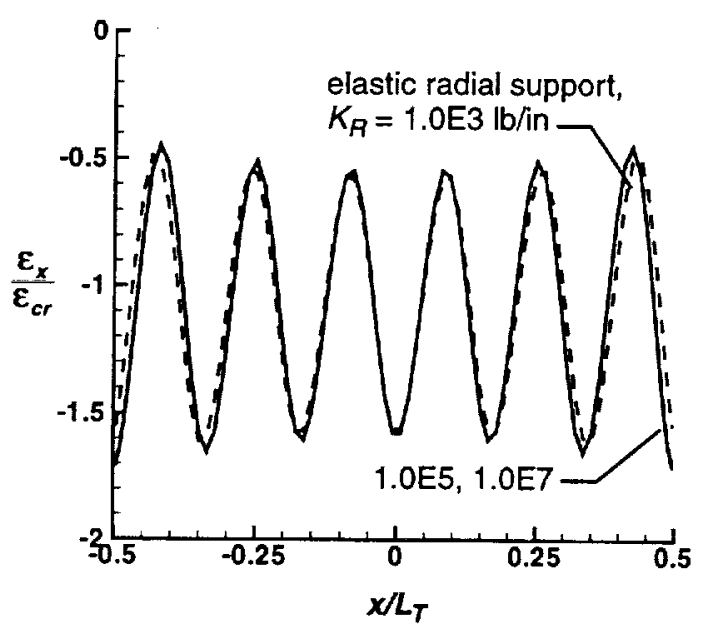

a) Geometrically perfect

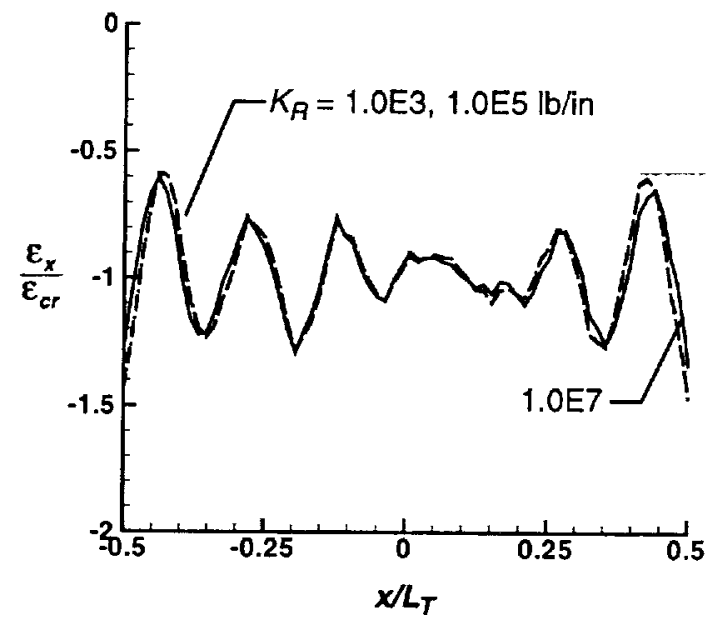

b) With measured imperfections

Fig. 23 Effects of elastic radial support conditions on the axial strain distribution of a compression-loaded orthotropic shell C1. 


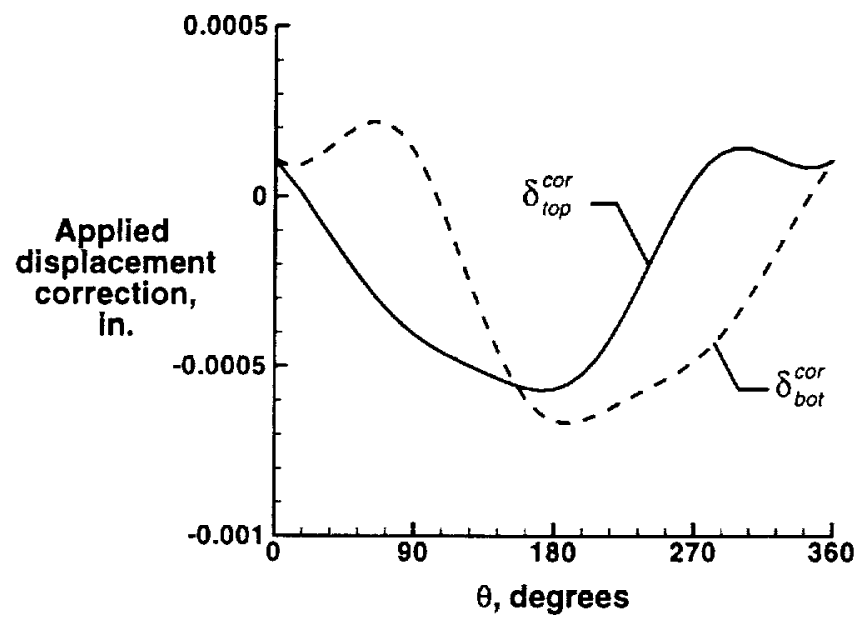

Fig. 24 Typical numerically predicted applied displacement corrections for the top $\left(\delta_{\text {top }}^{c o r}\right)$ and bottom $\left(\delta_{b o t}^{c o r}\right)$ specimen ends that are inferred from experimentally measured axial strains for quasi-isotropic shell C3.

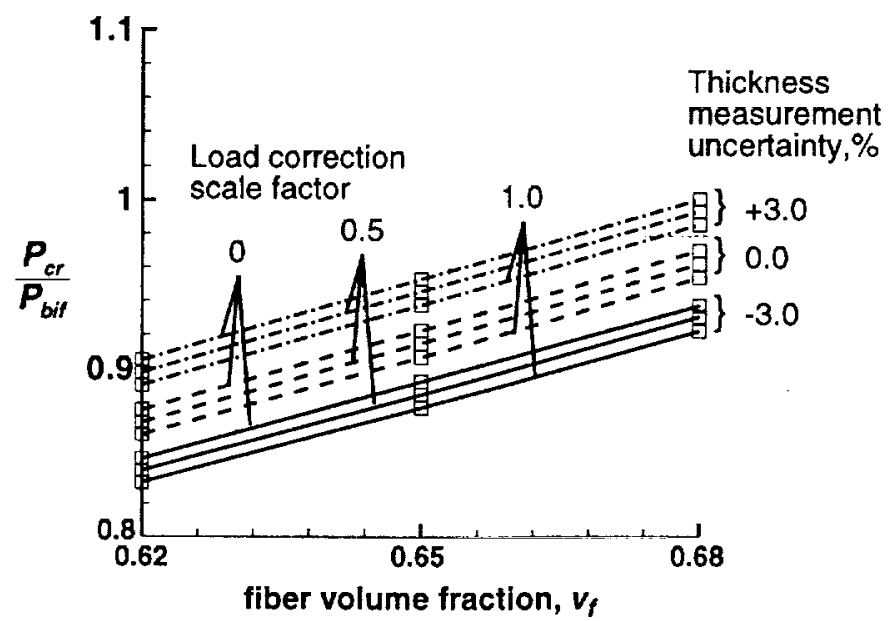

Fig. 25 Effects of variations in fiber volume fraction, measured shell-wall thickness, and applied load on the normalized buckling load of quasi-isotropic shell $\mathrm{C} 3$. 


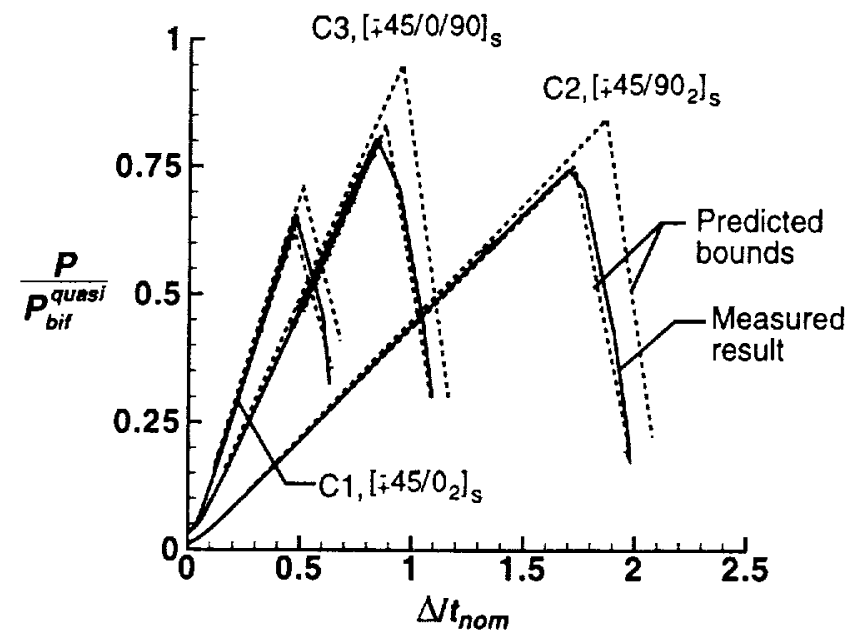

Fig. 26 Numerically predicted and experimentally measured load-end-shortening response curves; predicted results represent response bounds.
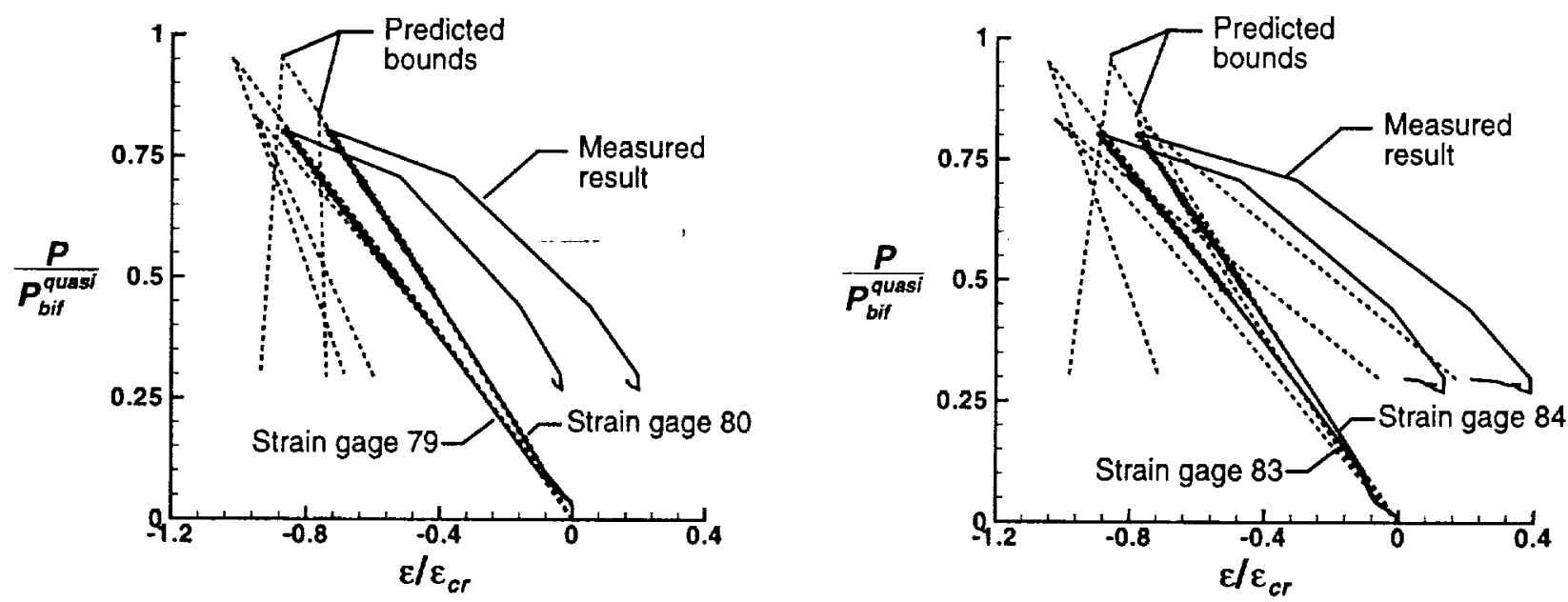

Fig. 27 Numerically predicted and experimentally measured load-strain response curves for quasi-isotropic shell C3; predicted results represent response bounds. 


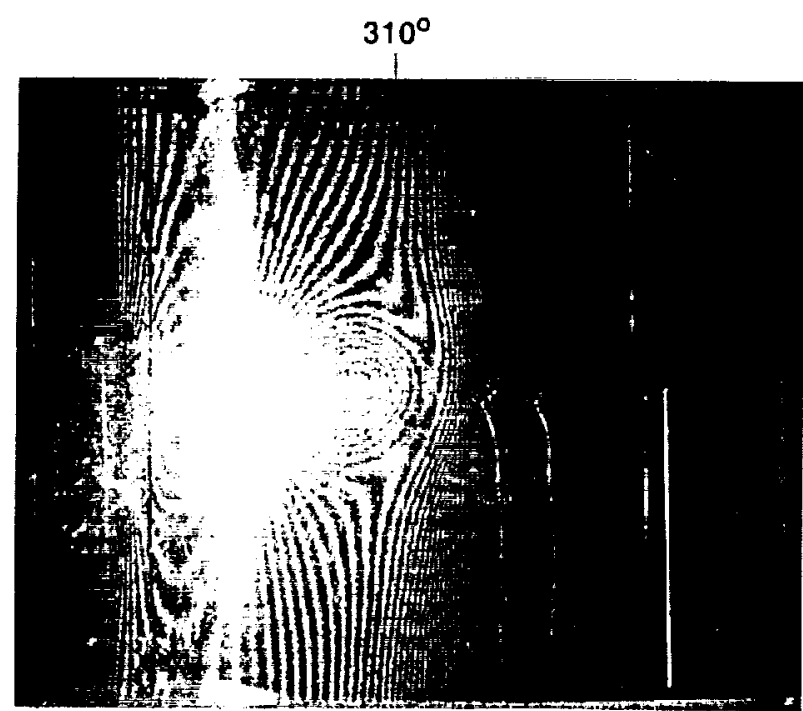

a) Observed post-collapse moiré fringe pattern.

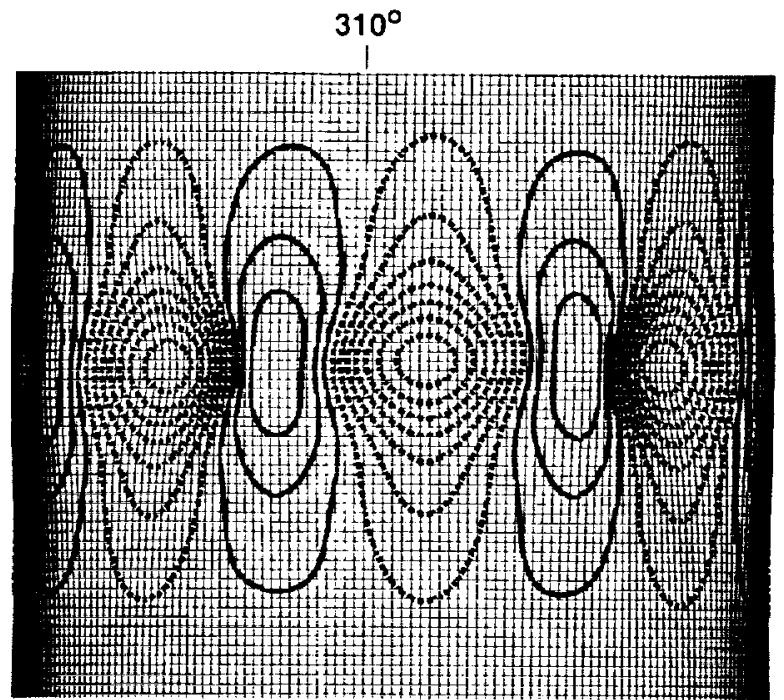

b) Numerically predicted post-collapse normal deformation response.

Fig. 28 Observed and predicted initial post-collapse normal displacements for specimen $\mathrm{C}$.

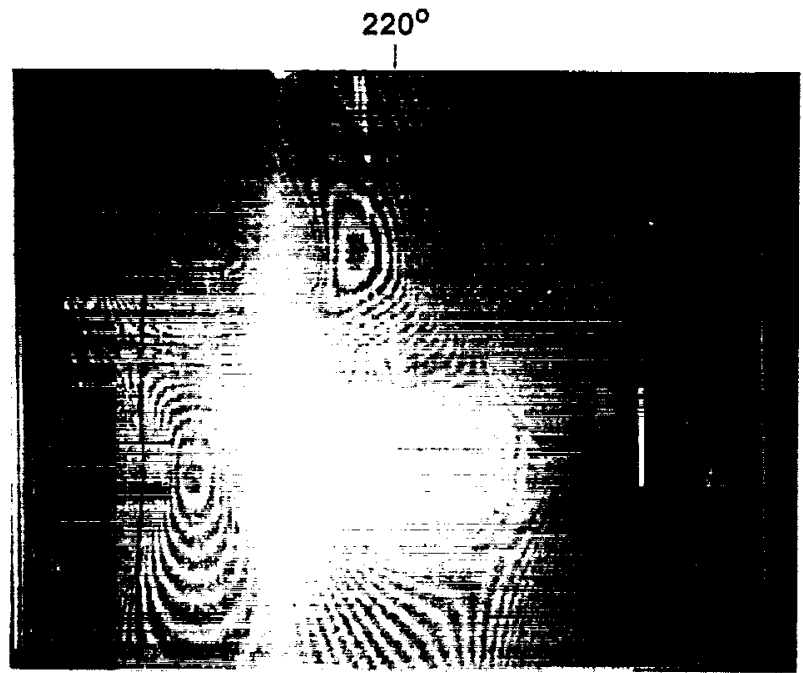

a) Observed post-collapse moiré fringe pattern.

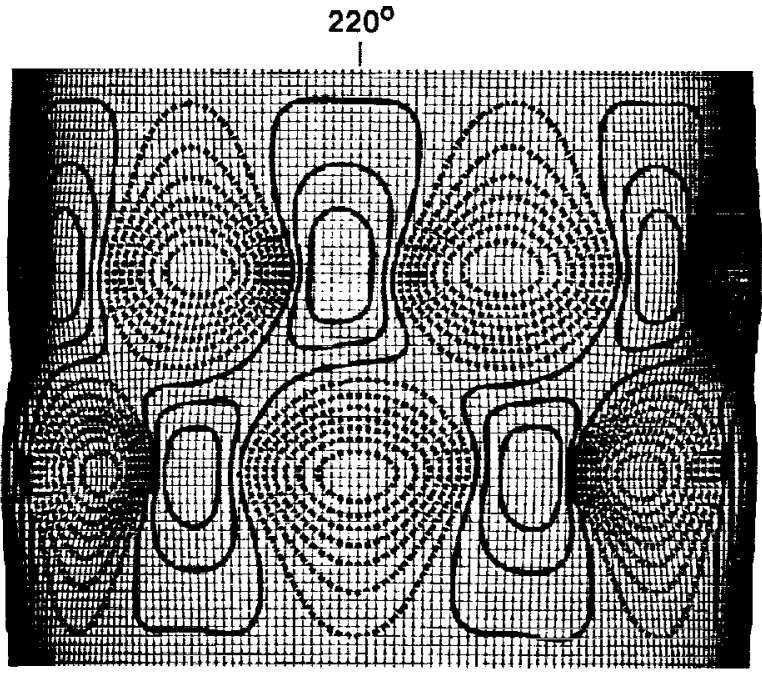

b) Numerically predicted post-collapse normal deformation response.

Fig. 29 Observed and predicted initial post-collapse normal displacements for specimen $\mathrm{C} 2$. 


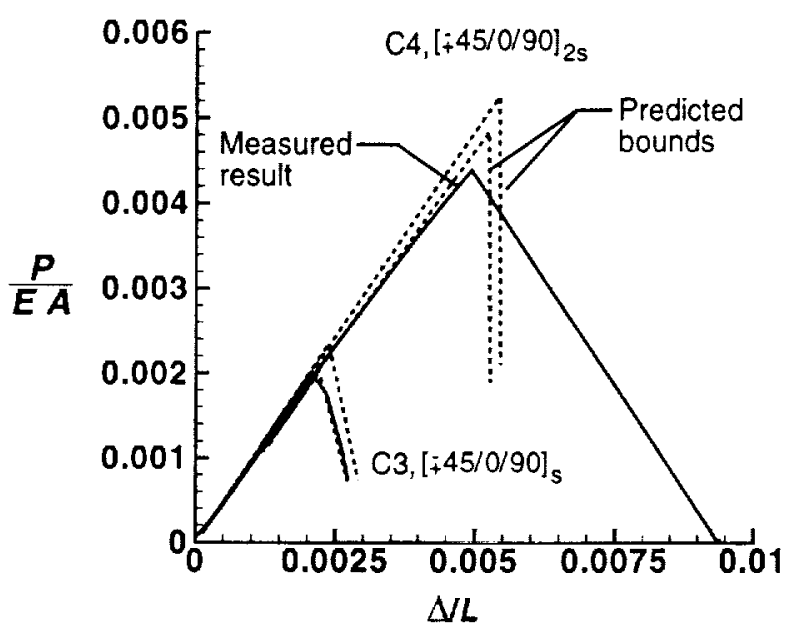

Fig. 30 Numerically predicted and experimentally measured load-end-shortening response curves for quasiisotropic shells $\mathrm{C} 3$ and $\mathrm{C4}$.

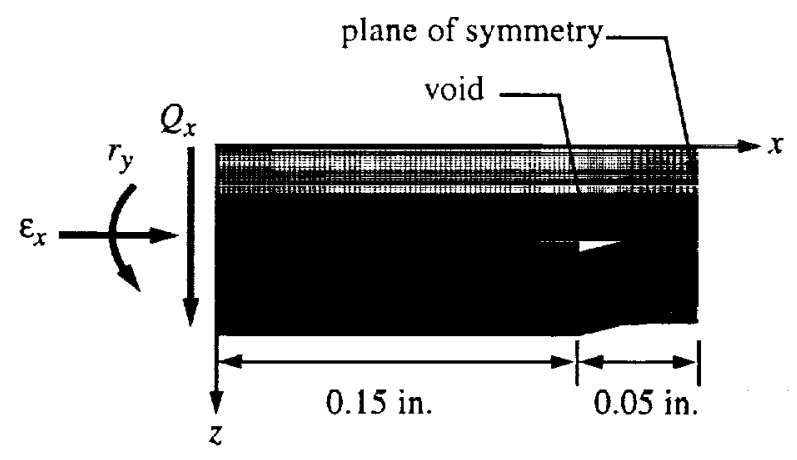

a) Typical two-dimensional model of a ply-gap feature in a shell wall subjected to combined loads.
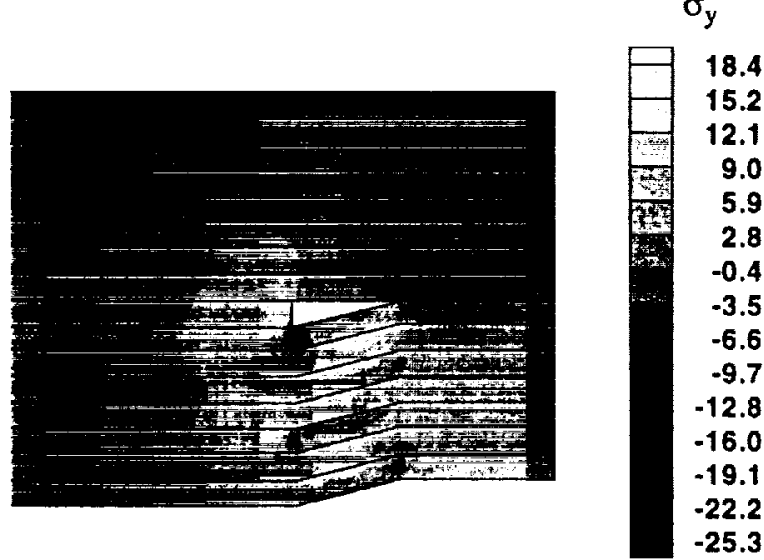

c) Transverse stress contours, $\sigma_{\mathrm{z}}, \mathrm{lbf} / \mathrm{in}^{2}$. $\sigma_{\mathrm{x}}$

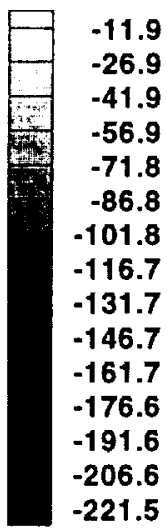

b) Axial stress contours, $\sigma_{\mathrm{x}}, \mathrm{lbf} / \mathrm{in}^{2}$.

$\sigma_{\mathrm{xy}}$

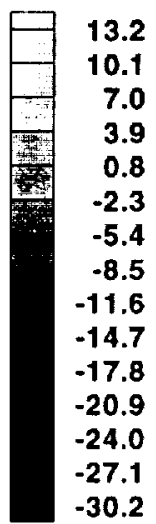

d) Transverse shear stress contours, $\sigma_{\mathrm{xz}}, \mathrm{Ibf} / \mathrm{in}^{2}$.

Fig. 31 Typical two-dimensional ply-gap model and numerically predicted through-the-thickness stress contours near a ply-gap in the sixteen-ply quasi-isotropic shell $\mathrm{C4}$. 


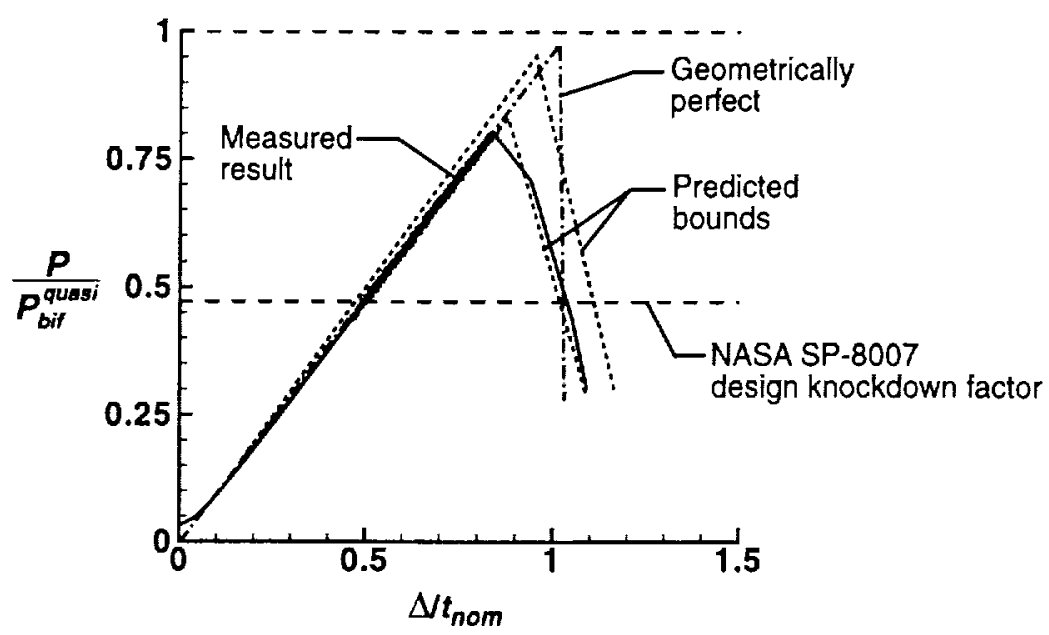

Fig. 32 Numerically predicted and experimentally measured load-strain response curves for quasi-isotropic shell C3. 